\title{
Kähler manifolds and transcendental techniques in algebraic geometry
}

\author{
Jean-Pierre Demailly
}

\begin{abstract}
Our goal is to survey some of the main advances which took place recently in the study of the geometry of projective or compact Kähler manifolds : very efficient new transcendental techniques, a better understanding of the geometric structure of cones of positive cohomology classes and of the deformation theory of Kähler manifolds, new results around the invariance of plurigenera and in the minimal model program.
\end{abstract}

Mathematics Subject Classification (2000). Primary 14C30; Secondary 32C17, 32C30, 32L20.

Keywords. Projective variety, Kähler manifold, Hodge theory, positive current, MongeAmpère equation, Lelong number, Chern connection, curvature, Bochner-Kodaira technique, Kodaira embedding theorem, Kähler cone, ample divisor, nef divisor, pseudoeffective cone, Neron-Severi group, $L^{2}$ estimates, vanishing theorem, Ohsawa-Takegoshi extension theorem, pluricanonical ring, invariance of plurigenera.

\section{Introduction}

Modern algebraic geometry is one the most intricate crossroads between various branches of mathematics : commutative algebra, complex analysis, global analysis on manifolds, partial differential equations, differential topology, symplectic geometry, number theory ... . This interplay has already been strongly emphasized by historical precursors, including Hodge, Kodaira, Hirzebruch and Grauert. Of course, there have been also fruitful efforts to establish purely algebraic foundations of the major results of algebraic geometry, and many prominent mathematicians such as Grothendieck, Deligne and Mumford stand out among the founders of this trend. The present contribution stands closer to the above mentioned wider approch ; its goal is to explain some recent applications of local and global complex analytic methods to the study of projective algebraic varieties.

A unifying theme is the concept of positivity : ample line bundles are characterized by the positivity of their curvature in the complex geometric setting (Kodaira [35]). Projective manifolds thus appear as a subclass of the class of compact Kähler manifolds, and their cohomological properties can be derived from the study of harmonic forms on Kähler manifolds (Hodge theory). In this vein, another central concept is the concept of positive current, which was introduced by P. Lelong during the 50's. By carefully studying the singularities and the intersection 
theory of such currents, we derive precise structure theorems for the Kähler cone and for the cone of effective divisors of arbitrary projective varieties ([5], [18]).

$L^{2}$ estimates for solutions of $\bar{\partial}$ equations are another crucial technique for proving vanishing theorems for the cohomology of vholomorphic vector bundles or sheaves. A combination of the Bochner-Kodaira differential geometric estimate with PDE techniques of Kohn, Hörmander and Andreotti-Vesentini led in the 60's to powerful existence theorems for $\bar{\partial}$-equations in hermitian vector bundles of positive curvature. A more recent and equally decisive outcome is the $L^{2}$ extension theorem by Ohsawa and Takegoshi [48] in 1987. Among applications, we have various forms of approximation theorems (closed positive $(1,1)$-currents can be approximated by algebraic divisors, and their singularities can be approximated by algebraic singularities). In the analytic setting, this turns out to be the key for the study of adjunction theory (generation properties of adjoint linear systems $K_{X}+L$, pluricanonical embeddings ...). As an illustration, we present a recent proof, adapted from work by Y.T. Siu [58], [59], S. Takayama [62] and M. Păun [52], of the deformation invariance of plurigenera $h^{0}\left(X_{t}, m K_{X_{t}}\right)$, for an arbitrary projective family $\left(X_{t}\right)$ of algebraic varieties.

\section{Basic concepts and results of complex geometry}

This section mostly contains only well-known definitions and results. However, we want to fix the notation and describe in detail our starting point.

2.1. Forms, currents, Kähler metrics. Let $X$ be a compact complex manifold and $n=\operatorname{dim}_{\mathbb{C}} X$. In any local holomorphic coordinate system $z=\left(z_{1}, \ldots, z_{n}\right)$, a differential form $u$ of type $(p, q)$ can be written as a sum $u(z)=\sum_{|J|=p,|K|=q} u_{J K}(z) d z_{J} \wedge d \bar{z}_{K}$ extended to all increasing multi-indices $J, K$ of length $p$, $q$, with the usual notation $d z_{J}=d z_{j_{1}} \wedge \ldots \wedge d z_{j_{p}}$. We are especially interested in positive currents of type $(p, p)$

$$
T=i^{p^{2}} \sum_{|J|=|K|=p} T_{J K}(z) d z_{J} \wedge d \bar{z}_{K} .
$$

Recall that a current is a differential form with distribution coefficients, and that a current is said to be positive if the distribution $\sum \lambda_{j} \bar{\lambda}_{k} T_{J K}$ is a positive real measure for all complex numbers $\lambda_{J}$ (which implies $T_{K J}=\bar{T}_{J K}$, hence $\bar{T}=T$ ). The coefficients $T_{J K}$ are then complex measures - and the diagonal ones $T_{J J}$ are positive (real) measures.

A current is said to be closed if $d T=0$ in the sense of distributions. Important examples of closed positive $(p, p)$-currents are currents of integration over codimension $p$ analytic cycles $[A]=\sum c_{j}\left[A_{j}\right]$ where the current $\left[A_{j}\right]$ is defined by duality as

$$
\left\langle\left[A_{j}\right], u\right\rangle=\int_{A_{j}} u_{\mid A_{j}}
$$


for every $(n-p, n-p)$ test form $u$ on $X$. Another important example of $(1,1)$ current is the Hessian form $T=i \partial \bar{\partial} \varphi$ of a plurisubharmonic function on an open set $\Omega \subset X$ (plurisubharmonic functions are upper semi-continuous functions satisfying the mean value inequality on complex analytic disc; they are characterized by positivity of $\left.i \sum \partial^{2} \varphi / \partial z_{j} \partial \bar{z}_{k} d z_{j} \wedge d \bar{z}_{k}\right)$. A Kähler metric on $X$ is a positive definite hermitian $(1,1)$-form

$$
\omega(z)=i \sum_{1 \leq j, k \leq n} \omega_{j k}(z) d z_{j} \wedge d \bar{z}_{k} \quad \text { such that } d \omega=0,
$$

with smooth coefficients. The manifold $X$ is said to be Kähler if it possesses at least one Kähler metric $\omega$. It is clear that every complex analytic and locally closed submanifold $X \subset \mathbb{P}_{\mathbb{C}}^{N}$ is Kähler (the restriction of the Fubini-Study metric $\omega_{F S}=\frac{i}{2 \pi} \log \left(\left|z_{0}\right|^{2}+\left|z_{1}\right|^{2}+\ldots+\left|z_{N}\right|^{2}\right)$ to $X$ is a Kähler metric. Especially projective algebraic varieties are Kähler.

2.2. Cohomology of compact Kähler manifolds. To every $d$-closed complex valued $k$-form or current $\alpha$ (resp. to every $\bar{\partial}$-closed complexe valued $(p, q)$ form or current $\alpha$ ) is associated its De Rham (resp. Dolbeault) cohomology class

$$
\{\alpha\} \in H^{p+q}(X, \mathbb{C}) \quad\left(\text { resp. } H^{p, q}(X, \mathbb{C})\right)
$$

This definition hides a nontrivial result, namely the fact that all cohomology groups involved (De Rham, Dolbeault, ...) can be defined either in terms of smooth forms or in terms of currents. In fact, if we consider the associated complexes of sheaves, forms and currents both provide acyclic resolutions of the same sheaf (locally constant functions, resp. holomorphic sections). One of the main results of Hodge theory, historically obtained by W.V.D. Hodge through the theory of harmonic forms, is the following fundamental

Theorem 2.1. Let $(X, \omega)$ be a compact Kähler manifold. Then there is a canonical isomorphism

$$
H^{k}(X, \mathbb{C})=\bigoplus_{p+q=k} H^{p, q}(X, \mathbb{C}),
$$

where each group $H^{p, q}(X, \mathbb{C})$ can ve viewed as the space of $(p, q)$-forms $\alpha$ which are harmonic with respect to $\omega$, i.e. $\Delta_{\omega} \alpha=0$.

Now, observe that every analytic cycle $A=\sum \lambda_{j} A_{j}$ of codimension $p$ with integral coefficients defines a cohomology class

$$
\{[A]\} \in H^{p, p}(X, \mathbb{C}) \cap H^{2 p}(X, \mathbb{Z}) /\{\text { torsion }\} \subset H^{p, p}(X, \mathbb{C}) \cap H^{2 p}(X, \mathbb{Q})
$$

where $H^{2 p}(X, \mathbb{Z}) /\{$ torsion $\} \subset H^{2 p}(X, \mathbb{Q}) \subset H^{2 p}(X, \mathbb{C})$ denotes the image of integral classes in complex cohomology. When $X$ is a projective algebraic manifold, this observation leads to the following statement, known as the Hodge conjecture (which was to become one of the famous seven Millenium problems of the Clay Mathematics Institute ...). 
Conjecture 2.2. Let $X$ be a projective algebraic manifold. Then the space of "Hodge classes" $H^{p, p}(X, \mathbb{C}) \cap H^{2 p}(X, \mathbb{Q})$ of type $(p, p)$ is generated by classes of algebraic cycles of codimension p with $\mathbb{Q}$-coefficients.

At present, not much is known to support the positive direction of the Hodge conjecture, not even the case of abelian varieties (i.e. projective algebraic complex tori $X=\mathbb{C} / \Lambda$ ) - which is the reason why several experts believe that the conjecture could eventually lead to a counterexample. There are however a number of cases where the cohomology algebra can be explicitly computed in terms of the geometry, and which do satisfy the conjecture: flag manifolds (Schubert cycles generate the cohomology ring), moduli spaces of stable or parabolic bundles over a general curve (I. Biswas and M.S. Narasimhan [2]).

In the Kähler case, the conjecture is trivially wrong as shown by a general complex torus possessing a line bundle with indefinite curvature. Moreover, by a recent result of C. Voisin [66], even a considerably weakened form of the conjecture - adding Chern classes of arbitrary coherent analytic sheaves to the pool of potential generators - is false for non projective complex tori:

Theorem 2.3. (C. Voisin [66]) There exists a 4-dimensional complex torus $X$ which possesses a non trivial Hodge class of degree 4, such that every coherent analytic sheaf $\mathcal{F}$ on $X$ satisfies $c_{2}(\mathcal{F})=0$.

The idea is to show the existence of a 4-dimensional complex torus $X=\mathbb{C}^{4} / \Lambda$ which does not contain any analytic subset of positive dimension, and such that the Hodge classes of degree 4 are perpendicular to $\omega^{n-2}$ for a suitable choice of the Kähler metric $\omega$. The lattice $\Lambda$ is explicitly found via a number theoretic construction of Weil based on the number field $\mathbb{Q}[i]$, also considered by S. Zucker [70]. The theorem of existence of Hermitian Yang-Mills connections for stable bundles combined with Lübke's inequality then implies $c_{2}(\mathcal{F})=0$ for every coherent sheaf $\mathcal{F}$ on the torus.

2.3. Fundamental $L^{2}$ existence theorems. Let $X$ be a complex manifold and $(E, h)$ a hermitian holomorphic vector bundle of rank $r$ over $X$. If $E_{\mid U} \simeq U \times \mathbb{C}^{r}$ is a local holomorphic trivialization, the hermitian product can be written as $\langle u, v\rangle={ }^{t} u H(z) \bar{v}$ where $H(z)$ is the hermitian matric of $h$ and $u, v \in E_{z}$. It is well known that there exists a unique "Chern connection" $D=D^{1,0}+D^{0,1}$ such that $D^{0,1}=\bar{\partial}$ and such that $D$ is compatible with the hermitian metric; in the given trivialization we have $D^{1,0} u=\bar{\partial} u+\Gamma^{1,0} \wedge u$ where $\Gamma^{1,0}=\bar{H}^{-1} \partial \bar{H}$, and its curvature operator $\Theta_{E, h}=D^{2}$ is the smooth section of $\Lambda^{1,1} T_{X}^{*} \otimes \operatorname{Hom}(E, E)$ given by $\Theta_{E, h}=\bar{\partial}\left(\bar{H}^{-1} \partial \bar{H}\right)$. If $E$ is of rank $r=1$, then it is customary to write $H(z)=e^{-\varphi(z)}$, and the curvature tensor then takes the simple expression $\Theta_{E, h}=\partial \bar{\partial} \varphi$. In that case, the first Chern class of $E$ is the cohomology class $c_{1}(E)=\left\{\frac{i}{2 \pi} \Theta_{E, h}\right\} \in H^{1,1}(X, \mathbb{C})$, which is also an integral class in $\left.H^{2}(X, \mathbb{Z})\right)$.

In case $(X, \omega)$ is a Kähler manifold, the bundles $\Lambda^{p, q} T_{X}^{*} \otimes E$ are equipped with the hermitian metric induced by $\Lambda^{p, q} \omega \otimes h$, and we have a Hilbert space of global $L^{2}$ sections over $X$ by integrating with respect to the Kähler volume form 
$d V_{\omega}=\omega^{n} / n$ !. If $A, B$ are differential operators acting on $L^{2}$ space of sections (in general, they are just closed and densely defined operators), we denote by $A^{*}$ the formal adjoint of $A$, and by $[A, B]=A B-(-1)^{\operatorname{deg} A \operatorname{deg} B} B A$ the usual commutator bracket of operators. The fundamental operator $\Lambda_{\omega}$ of Kähler geometry is the adjoint of the wedge multiplication operator $u \mapsto \omega \wedge u$.

In this context, we have the following fundamental existence theorems for $\bar{\partial}-$ equations, which is the culmination of several decades of work by Bochner [3], Kodaira [35], Kohn [36], Andreotti-Vesentini [1], Hörmander [25], Skoda [60], OhsawaTakegoshi [48] (and many others). The proofs always proceed through differential geometric inequalities relating the Laplace-Beltrami operators with the curvature (Bochner-Kodaira identities and inequalities). The most basic result is the $L^{2}$ existence theorem for solutions of $\bar{\partial}$-equations.

Theorem 2.4. ([1], see also [10]) Let $(X, \omega)$ be a Kähler manifold which is "complete" in the sense that it possesses a geodesically complete Kähler metric $\widetilde{\omega}$. Let $E$ be a hermitian holomorphic vector bundle of rank $r$ over $X$, and assume that the curvature operator $A_{E, h, \omega}^{p, q}=\left[i \Theta_{E, h}, \Lambda_{\omega}\right]$ is positive definite everywhere on $\Lambda^{p, q} T_{X}^{\star} \otimes E, q \geq 1$. Then for any form $g \in L^{2}\left(X, \Lambda^{p, q} T_{X}^{\star} \otimes E\right)$ satisfying $\bar{\partial} g=0$ and $\int_{X}\left\langle\left(A_{E, h, \omega}^{p, q}\right)^{-1} g, g\right\rangle d V_{\omega}<+\infty$, there exists $f \in L^{2}\left(X, \Lambda^{p, q-1} T_{X}^{\star} \otimes E\right)$ such that $\bar{\partial} f=g$ and

$$
\int_{X}|f|^{2} d V_{\omega} \leq \int_{X}\left\langle\left(A_{E, h, \omega}^{p, q}\right)^{-1} g, g\right\rangle d V_{\omega}
$$

It is thus of crucial importance to study conditions under which the operator $A_{E, h, \omega}^{p, q}$ is positive definite. An easier case is when $E$ is a line bundle. Then we denote by $\gamma_{1}(z) \leq \ldots \leq \gamma_{n}(z)$ the eigenvalues of the real $(1,1)$-form $i \Theta_{E, h}(z)$ with respect to the metric $\omega(z)$ at each point. A straightforward calculation shows that

$$
\left\langle A_{E, h, \omega}^{p, q} u, u\right\rangle=\sum_{|J|=p,|K|=q}\left(\sum_{k \in K} \gamma_{k}-\sum_{j \in \mathrm{C} J} \gamma_{j}\right)\left|u_{J K}\right|^{2} .
$$

In particular, for $(n, q)$-forms the negative sum $-\sum_{j \in \mathrm{C} J} \gamma_{j}$ disappears and we have

$$
\left\langle A_{E, h, \omega}^{n, q} u, u\right\rangle \geq\left(\gamma_{1}+\ldots+\gamma_{q}\right)|u|^{2}, \quad\left\langle\left(A_{E, h, \omega}^{n, q}\right)^{-1} u, u\right\rangle \leq\left(\gamma_{1}+\ldots+\gamma_{q}\right)^{-1}|u|^{2}
$$

provided the line bundle $(E, h)$ has positive definite curvature. Therefore $\bar{\partial}$ equations can be solved for all $L^{2}(n, q)$-forms with $q \geq 1$, and this is the major reason why vanishing results for $H^{q}$ cohomology groups are usually obtained for sections of the "adjoint line bundle" $\widetilde{E}=K_{X} \otimes E$, where $K_{X}=\Lambda^{n} T_{X}^{*}=\Omega_{X}^{n}$ is the "canonical bundle" of $X$, rather than for $E$ itself. Especially, if $X$ is compact (or weakly pseudoconvex) and $i \Theta_{E, h}>0$, then $H^{q}\left(X, K_{X} \otimes E\right)=0$ for $q \geq 1$ (Kodaira), and more generally $H^{p, q}(X, E)=0$ for $p+q \geq n+1$ (Kodaira-Nakano, take $\omega=i \Theta_{E, h}$, in which case $\gamma_{j} \equiv 1$ for all $j$ and $\left.\sum_{k \in K} \gamma_{k}-\sum_{j \in \mathrm{C} J} \gamma_{j}=p+q-n\right)$.

As shown in [10], Theorem 2.4 still holds true in that case when $h$ is a singular hermitian metric, i.e. a metric whose weights $\varphi$ are arbitrary locally integrable functions, provided that the curvature is $(E, h)$ is positive in the sense of currents (i.e., 
the weights $\varphi$ are strictly plurisubharmonic). This implies the well-known Nadel vanishing theorem ([42], [12], [15]), a generalization of the Kawamata-Viehweg vanishing theorem [28], [65].

Theorem 2.5. (Nadel) Let $(X, \omega)$ be a compact (or weakly pseudoconvex) Kähler manifold, and $(L, h)$ a singular hermitian line bundle such that $\Theta_{L, h} \geq \varepsilon \omega$ for some $\varepsilon>0$. Then $H^{q}\left(X, K_{X} \otimes L \otimes \mathcal{I}(h)\right)=0$ for $q \geq 1$, where $\mathcal{I}(h)$ is the multiplier ideal sheaf of $h$, namely the sheaf of germs of holomorphic functions $f$ on $X$ such that $|f|^{2} e^{-\varphi}$ is locally integrable with respect to the local weights $h=e^{-\varphi}$.

It is well known that Theorems 2.4 and 2.5, more specifically, its "singular hermitian" version, imply almost all other fundamental vanishing or existence theorems of algebraic geometry, as well as their analytic counterparts in the framework of Stein manifolds (general solution of the Levi problem by Grauert), see e.g. Demailly [16] for a recent account. In particular, one gets as a consequence the Kodaira embedding theorem [35].

Theorem 2.6. Let $X$ be a compact complex $n$-dimensional manifold. Then the following properties are equivalent.

(i) $X$ can be embedded in some projective space $\mathbb{P}_{\mathbb{C}}^{N}$ as a closed analytic submanifold (and such a submanifold is automatically algebraic by Chow's thorem).

(ii) $X$ carries a hermitian holomorphic line bundle $(L, h)$ with psoitive definite smooth curvature form $i \Theta_{L, h}>0$.

(iii) $X$ possesses a Hodge metric, i.e., a Kähler metric $\omega$ such that $\{\omega\} \in H^{2}(X, \mathbb{Z})$.

If property (ii) holds true, then for $m \geq m_{0} \gg 1$ the multiple $L^{\otimes m}$ is very ample, namely we have an embedding given by the linear system $V=H^{0}\left(X, L^{\otimes m}\right)$ of sections,

$$
\Phi_{L^{\otimes m}}: X \longrightarrow P\left(V^{*}\right), \quad z \mapsto H_{z}=\{\sigma \in V ; \sigma(z)=0\} \subset V,
$$

and $L^{\otimes m} \simeq \Phi_{L \otimes m}^{*} \mathcal{O}(1)$ is the pull-back of the canonical bundle on $P\left(V^{*}\right)$.

Another fundamental existence theorem is the $L^{2}$-extension result by OhsawaTakegoshi [48]. Many different versions and generalizations have been given in recent years [43], [44], [45], [46], [47]. Here is another one, due to Manivel [40], which is slightly less general but simpler to state.

Theorem 2.7. (Ohsawa-Takegoshi [48], Manivel [40]) Let $X$ be a compact or weakly pseudoconvex n-dimensional complex manifold equipped with a Kähler metric $\omega$, let $L$ (resp. E) be a hermitian holomorphic line bundle (resp. a hermitian holomorphic vector bundle of rank $r$ over $X$ ), and $s$ a global holomorphic section of $E$. Assume that $s$ is generically transverse to the zero section, and let

$$
Y=\left\{x \in X ; s(x)=0, \Lambda^{r} d s(x) \neq 0\right\}, \quad p=\operatorname{dim} Y=n-r .
$$

Moreover, assume that the $(1,1)$-form $i \Theta(L)+r i \partial \bar{\partial} \log |s|^{2}$ is semipositive and that there is a continuous function $\alpha \geq 1$ such that the following two inequalities hold everywhere on $X$ : 
(i) $i \Theta(L)+r i \partial \bar{\partial} \log |s|^{2} \geq \alpha^{-1} \frac{\{i \Theta(E) s, s\}}{|s|^{2}}$,

(ii) $|s| \leq e^{-\alpha}$.

Then for every holomorphic section $f$ over $Y$ of the adjoint line bundle $\widetilde{L}=K_{X} \otimes L$ (restricted to $Y$ ), such that $\int_{Y}|f|^{2}\left|\Lambda^{r}(d s)\right|^{-2} d V_{\omega}<+\infty$, there exists a holomorphic extension $F$ of $f$ over $X$, with values in $\widetilde{L}$, such that

$$
\int_{X} \frac{|F|^{2}}{|s|^{2 r}(-\log |s|)^{2}} d V_{X, \omega} \leq C_{r} \int_{Y} \frac{|f|^{2}}{\left|\Lambda^{r}(d s)\right|^{2}} d V_{Y, \omega},
$$

where $C_{r}$ is a numerical constant depending only on $r$.

The proof actually shows that the extension theorem holds true as well for $\bar{\partial}$-closed $(0, q)$-forms with values in $\widetilde{L}$, of which the stated theorem is the special case $q=0$.

There are several other important $L^{2}$ existence theorems. One of them is Skoda's criterion for the surjectivity of holomorphic bundle morphisms - more concretely, a Bezout type division theorem for holomorphic function. It can be derived either from Theorem 2.4 on $\bar{\partial}$-equations through sharp curvature calculations (this is Skoda's original approch in [60]), or as a consequence of the above extension theorem 2.7 (see Ohsawa [46]).

2.4. Positive cones. We now introduce some further basic objects of projective or Kähler geometry, namely cones of positive cohomology classes.

Definition 2.8. Let $X$ be a compact Kähler manifold and $H^{1,1}(X, \mathbb{R})$ the space of real $(1,1)$ cohomology classes.

(i) The Kähler cone is the set $\mathcal{K} \subset H^{1,1}(X, \mathbb{R})$ of cohomology classes $\{\omega\}$ of Kähler forms. This is clearly an open convex cone.

(ii) The pseudo-effective cone is the set $\mathcal{E} \subset H^{1,1}(X, \mathbb{R})$ of cohomology classes $\{T\}$ of closed positive currents of type $(1,1)$. This is a closed convex cone (as follows from the weak compactness property of bounded sets of positive measures or currents).

It is follows from this definition that $\overline{\mathcal{K}} \subset \mathcal{E}$. In general the inclusion is strict. To see this, it is enough to observe that a Kähler class $\{\alpha\}$ satisfies $\int_{Y} \alpha^{p}>0$ for every $p$-dimensional analytic set. On the other hand, if $X$ is the surface obtained by blowing-up $\mathbb{P}^{2}$ in one point, then the exceptional divisor $E \simeq \mathbb{P}^{1}$ has a cohomology class $\{\alpha\}$ such that $\int_{E} \alpha=E^{2}=-1$, hence $\{\alpha\} \notin \overline{\mathcal{K}}$, although $\{\alpha\}=\{[E]\} \in \mathcal{E}$.

In case $X$ is projective, it is interesting to consider also the algebraic analogues of our "transcendental cones" $\mathcal{K}$ and $\mathcal{E}$, which consist of suitable integral divisor classes. Since the cohomology classes of such divisors live in $H^{2}(X, \mathbb{Z})$, we are led to introduce the Neron-Severi lattice and the associated Neron-Severi space

$$
\mathrm{NS}(X):=H^{1,1}(X, \mathbb{R}) \cap\left(H^{2}(X, \mathbb{Z}) /\{\text { torsion }\}\right),
$$




$$
\mathrm{NS}_{\mathbb{R}}(X):=\mathrm{NS}(X) \otimes_{\mathbb{Z}} \mathbb{R}
$$

All classes of real divisors $D=\sum c_{j} D_{j}, c_{j} \in \mathbb{R}$, lie by definition in $\operatorname{NS}_{\mathbb{R}}(X)$. Notice that the integral lattice $H^{2}(X, \mathbb{Z}) /\{$ torsion $\}$ need not hit at all the subspace $H^{1,1}(X, \mathbb{R}) \subset H^{2}(X, \mathbb{R})$ in the Hodge decomposition, hence in general the Picard number, defined as

$$
\rho(X)=\operatorname{rank}_{\mathbb{Z}} \mathrm{NS}(X)=\operatorname{dim}_{\mathbb{R}} \mathrm{NS}_{\mathbb{R}}(X)
$$

satisfies $\rho(X) \leq h^{1,1}=\operatorname{dim}_{\mathbb{R}} H^{1,1}(X, \mathbb{R})$, but the equality can be strict (actually, it is well known that a generic complex torus $X=\mathbb{C}^{n} / \Lambda$ satisfies $\rho(X)=0$ and $\left.h^{1,1}=n^{2}\right)$. In order to deal with the case of algebraic varieties we introduce

$$
\mathcal{K}_{\mathrm{NS}}=\mathcal{K} \cap \mathrm{NS}_{\mathbb{R}}(X), \quad \mathcal{E}_{\mathrm{NS}}=\mathcal{E} \cap \mathrm{NS}_{\mathbb{R}}(X) .
$$

A very important fact is that the "Neron-Severi part" of any of the open or closed transcendental cones $\mathcal{K}, \mathcal{E}, \overline{\mathcal{K}}, \mathcal{E}^{\circ}$ is algebraic, i.e. can be characterized in simple algebraic terms.

Theorem 2.9. Let $X$ be a projective manifold. Then

(i) $\mathcal{E}_{\mathrm{NS}}$ is the closure of the cone generated by classes of effective divisors, i.e. divisors $D=\sum c_{j} D_{j}, c_{j} \in \mathbb{R}_{+}$.

(ii) $\mathcal{K}_{\mathrm{NS}}$ is the open cone generated by classes of ample (or very ample) divisors $A$ (Recall that a divisor $A$ is said to be very ample if the linear system $H^{0}(X, \mathcal{O}(A))$ provides an embedding of $X$ in projective space).

(iii) The interior $\mathcal{E}_{\mathrm{NS}}^{\circ}$ is the cone generated by classes of big divisors, namely divisors $D$ such that $h^{0}(X, \mathcal{O}(k D)) \geq c k^{\operatorname{dim} X}$ for $k$ large.

(iv) The closed cone $\overline{\mathcal{K}}_{\mathrm{NS}}$ consists of the closure of the cone generated by nef divisors $D$ (or nef line bundles $L$ ), namely effective integral divisors $D$ such that $D \cdot C \geq 0$ for every curve $C$.

By extension, we will say that $\overline{\mathcal{K}}$ is the cone of nef $(1,1)$-cohomology classes (even though they are not necessarily integral).

Sketch of proof. (see also [13] for more details). If we denote by $\mathcal{K}_{\text {alg }}$ the open cone generated by ample divisors, resp. by $\mathcal{E}_{\text {alg }}$ the closure of the cone generated by effective divisors, we have $K_{\text {alg }} \subset K_{\mathrm{NS}}, \mathcal{E}_{\text {alg }} \subset \mathcal{E}_{\mathrm{NS}}$, and clearly the interesting part lies in the converse inclusions. The inclusion $K_{\mathrm{NS}} \subset \mathcal{K}_{\text {alg }}$ is equivalent to the Kodaira embedding theorem : if a rational class $\{\alpha\}$ is in $\mathcal{K}$, then some multiple of $\{\alpha\}$ is the first Chern class of a hermitian line bundle $L$ whose curvature form is Kähler. Therefore $L$ is ample and $\{\alpha\} \in \mathcal{K}_{\text {alg }}$; property (ii) follows.

Similarly, if we take a rational class $\{\alpha\} \in \mathcal{E}_{\mathrm{NS}}^{\circ}$, then we still have $\{\alpha-\varepsilon \omega\} \in \mathcal{E}_{\mathrm{NS}}^{\circ}$ by subtracting a small multiple $\varepsilon \omega$ of a Kähler class, hence $\alpha-\varepsilon \omega \equiv T \geq 0$ for some positive current $T$. Therefore some multiple $\left\{m_{0} \alpha\right\}$ is the first Chern class of a hermitian line bundle $(L, h)$ with curvature current $T$

$$
\Theta_{L, h}:=-\frac{i}{2 \pi} i \partial \bar{\partial} \log h=m_{0}(T+\varepsilon \omega) \geq m_{0} \varepsilon \omega .
$$


Theorem 2.4 on $L^{2}$ estimates for $\bar{\partial}$-equations then shows that large multiples $L^{\otimes k}$ admit a large number of sections, hence $L^{\otimes k}$ can be represented by a big divisor. This implies (iii) and also that $\mathcal{E}_{\mathrm{NS}}^{\circ} \subset \mathcal{E}_{\text {alg. }}$. Therefore $\mathcal{E}_{\mathrm{NS}} \subset \mathcal{E}_{\text {alg }}$ by passing to the closure; (i) follows. The statement (iv) about nef divisors follows e.g. from Klaiman [34] and Hartshorne [24], since every nef divisor is a limit of a sequence of ample rational divisors.

As a natural extrapolation of the algebraic situation, we say that $\overline{\mathcal{K}}$ is the cone of nef (1,1)-cohomology classes (even though these classes are not necessarily integral). Property 2.9 (i) also explains the terminology used for the pseudoeffective cone.

\subsection{Approximation of currents and Zariski decomposition.} Let $X$ be compact Kähler manifold and let $\alpha \in \mathcal{E}^{\circ}$ be in the interior of the pseudo-effective cone. In analogy with the algebraic context, such a class $\alpha$ is called "big", and it can then be represented by a Kähler current T, i.e. a closed positive $(1,1)$-current $T$ such that $T \geq \delta \omega$ for some smooth hermitian metric $\omega$ and a constant $\delta \ll 1$. Notice that the latter definition of a Kähler current makes sense even if $X$ is an arbitrary (non necessarily Kähler) compact complex manifold.

Theorem 2.10. (Demailly [14], Boucksom [4], 3.1.24) If $T$ is a Kähler current on a compact complex manifold $X$, then one can write $T=\lim T_{m}$ for a sequence of Kähler currents $T_{m}$ in the same cohomology class as $T$, which have logarithmic poles and coefficients in $\frac{1}{m} \mathbb{Z}$. This means that there are modifications $\mu_{m}: \widetilde{X}_{m} \rightarrow X$ such that

$$
\mu_{m}^{\star} T_{m}=\left[E_{m}\right]+\beta_{m}
$$

where $E_{m}$ is an effective $\mathbb{Q}$-divisor on $\tilde{X}_{m}$ with coefficients in $\frac{1}{m} \mathbb{Z}\left(E_{m}\right.$ is the "fixed part" and $\beta_{m}$ a closed semi-positive form, the "movable part").

Proof. We just recall the main idea and refer to [14] for details. Locally we can write $T=i \partial \bar{\partial} \varphi$ for some strictly plurisubharmonic potential $\varphi$ on $X$. The approximating potentials $\varphi_{m}$ of $\varphi$ are defined as

$$
\varphi_{m}(z)=\frac{1}{2 m} \log \sum_{\ell}\left|g_{\ell, m}(z)\right|^{2}
$$

where $\left(g_{\ell, m}\right)$ is a Hilbert basis of the space $\mathcal{H}(\Omega, m \varphi)$ of holomorphic functions which are $L^{2}$ with respect to the weight $e^{-2 m \varphi}$. The Ohsawa-Takegoshi $L^{2}$ extension theorem 2.7 (applied to extension from a single isolated point) implies that there are enough such holomorphic functions, and thus $\varphi_{m} \geq \varphi-C / m$. On the other hand $\varphi=\lim _{m \rightarrow+\infty} \varphi_{m}$ by a Bergman kernel trick and by the mean value inequality.

The Hilbert basis $\left(g_{\ell, m}\right)$ is also a family of local generators of the globally defined multiplier ideal sheaf $\mathcal{I}(m T)=\mathcal{I}(m \varphi)$. The modification $\mu_{m}: \widetilde{X}_{m} \rightarrow X$ is obtained by blowing-up this ideal sheaf, so that

$$
\mu_{m}^{\star} \mathcal{I}(m T)=\mathcal{O}\left(-m E_{m}\right)
$$


for some effective $\mathbb{Q}$-divisor $E_{m}$ with normal crossings on $\widetilde{X}_{m}$. Now, we set $T_{m}=$ $i \partial \bar{\partial} \varphi_{m}$ and $\beta_{m}=\mu_{m}^{*} T_{m}-\left[E_{m}\right]$. Then $\beta_{m}=i \partial \bar{\partial} \psi_{m}$ where

$$
\psi_{m}=\frac{1}{2 m} \log \sum_{\ell}\left|g_{\ell, m} \circ \mu_{m} / h\right|^{2} \quad \text { locally on } \widetilde{X}_{m}
$$

and $h$ is a generator of $\mathcal{O}\left(-m E_{m}\right)$, and we see that $\beta_{m}$ is a smooth semi-positive form on $\widetilde{X}_{m}$. The construction can be made global by using a gluing technique, e.g. via partitions of unity.

Remark 2.11. The more familiar algebraic analogue would be to take $\alpha=c_{1}(L)$ with a big line bundle $L$ and to blow-up the base locus of $|m L|, m \gg 1$, to get a $\mathbb{Q}$-divisor decomposition

$$
\mu_{m}^{\star} L \sim E_{m}+D_{m}, \quad E_{m} \text { effective, } D_{m} \text { free. }
$$

Such a blow-up is usually referred to as a "log resolution" of the linear system $|m L|$, and we say that $E_{m}+D_{m}$ is an approximate Zariski decomposition of $L$. We will also use this terminology for Kähler currents with logarithmic poles.

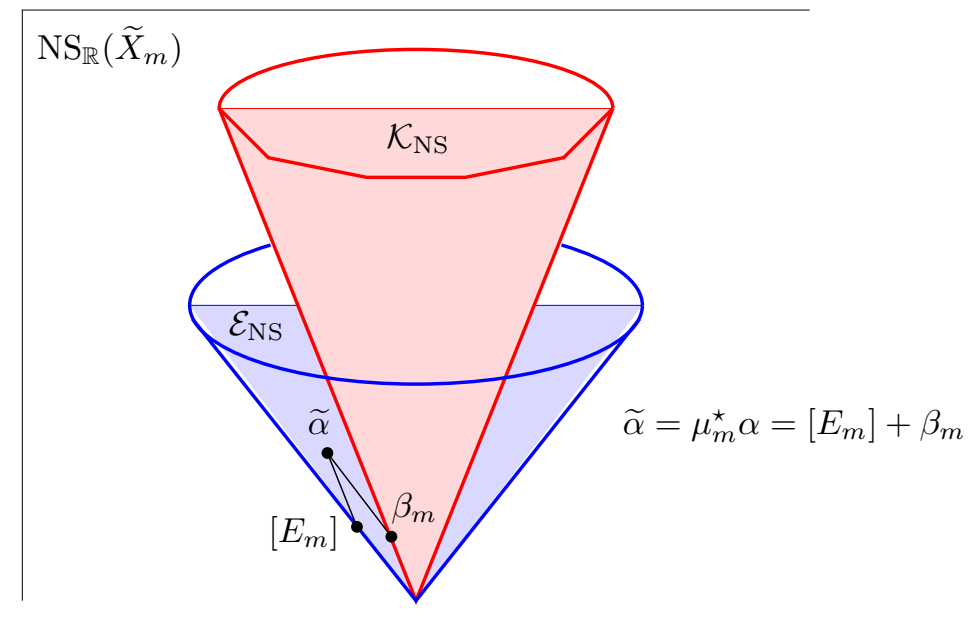

In the above construction, $\beta_{m}$ is not just semi-positive, it is even positive definite on tangent vectors which are not mapped to 0 by the differential $d \mu_{m}$, in particular $\beta_{m}$ is positive definite outside the exceptional divisor. However, if $E$ is the exceptional divisor of the blow-up along a smooth centre $Y \subset X$, then $\mathcal{O}(-E)$ is relatively ample with respect to the blow-up map $\pi$, hence the negative current $-[E]$ is cohomologous to a smooth form $\theta_{E}$ which is positive along the fibers of $\pi$. As a consequence, we can slightly perturb the decomposition of $\widetilde{\alpha}$ by increasing multiplicities in the components of $E_{m}$ and adding recursively to $\beta_{m}$ small multiples $\varepsilon_{E} \theta_{E}$ in such a way that $\widetilde{\beta}_{m}+\sum \varepsilon_{E} \theta_{E}$ becomes a Kähler metric on $\widetilde{X}_{m}$. This in 
turn implies that $\widetilde{X}_{m}$ is Kähler and we thus get the following characterization of the Fujiki class $\mathcal{C}$ of compact complex manifolds which are bimeromorphic to Kähler manifolds:

Corollary 2.12. A compact complex manifold is bimeromorphic to a Kähler manifold (or equivalently, dominated by a Kähler manifold) if and only if it carries a Kähler current $T$.

\section{Numerical characterization of the Kähler cone}

We describe here the main results obtained in Demailly-Păun [18]. The upshot is that the Kähler cone depends only on the intersection product of the cohomology ring, the Hodge structure and the homology classes of analytic cycles. More precisely, we have:

Theorem 3.1. Let $X$ be a compact Kähler manifold. Let $\mathcal{P}$ be the set of real $(1,1)$ cohomology classes $\{\alpha\}$ which are numerically positive on analytic cycles, i.e. such that $\int_{Y} \alpha^{p}>0$ for every irreducible analytic set $Y$ in $X, p=\operatorname{dim} Y$. Then the Kähler cone $\mathcal{K}$ of $X$ is one of the connected components of $\mathcal{P}$.

Corollary 3.2. If $X$ is projective algebraic, then $\mathcal{K}=\mathcal{P}$.

These results (which are new even in the projective case) can be seen as a generalization of the well-known Nakai-Moishezon criterion. Recall that the NakaiMoishezon criterion provides a necessary and sufficient criterion for a line bundle to be ample: a line bundle $L \rightarrow X$ on a projective algebraic manifold $X$ is ample if and only if

$$
L^{p} \cdot Y=\int_{Y} c_{1}(L)^{p}>0,
$$

for every algebraic subset $Y \subset X, p=\operatorname{dim} Y$.

It turns out that the numerical conditions $\int_{Y} \alpha^{p}>0$ also characterize arbitrary transcendental Kähler classes when $X$ is projective: this is precisely the meaning of Cor. 3.2.

Example 3.3. The following example shows that the cone $\mathcal{P}$ need not be connected (and that the components of $\mathcal{P}$ need not be convex, either). Consider for instance a complex torus $X=\mathbb{C}^{n} / \Lambda$. It is well-known that a generic torus $X$ does not possess any analytic subset except finite subsets and $X$ itself. In that case, the numerical positivity is expressed by the single condition $\int_{X} \alpha^{n}>0$. However, on a torus, $(1,1)$-classes are in one-to-one correspondence with constant hermitian forms $\alpha$ on $\mathbb{C}^{n}$. Thus, for $X$ generic, $\mathcal{P}$ is the set of hermitian forms on $\mathbb{C}^{n}$ such that $\operatorname{det}(\alpha)>0$, and Theorem 3.1 just expresses the elementary result of linear algebra saying that the set $\mathcal{K}$ of positive definite forms is one of the connected components of the open set $\mathcal{P}=\{\operatorname{det}(\alpha)>0\}$ of hermitian forms of positive determinant (the other components, of course, are the sets of forms of signature $(p, q)$, $p+q=n, q$ even. They are not convex when $p>0$ and $q>0)$. 
Sketch of proof of Theorem 3.1 and Cor. 3.2. As is well known, the singularities of a closed positive current $T$ can be measured by its Lelong numbers

$$
\nu(T, x)=\liminf _{z \rightarrow x} \frac{\varphi(z)}{\log |z-x|},
$$

where $T=\frac{i}{\pi} \partial \bar{\partial} \varphi$ near $x$. A fundamental theorem of Siu Siu [56] states that the Lelong sublevel sets $E_{c}(T):=\{x \in X ; \nu(T, x) \geq c\}$ are analytic sets for every $c>0$ (this fact can nowadays be derived in a rather straightforward manner from the approximation theorem 2.10). The crucial steps of the proof of Theorem 3.1 are contained in the following statements.

Proposition 3.4. (Păun [49], [50]) Let X be a compact complex manifold (or more generally a compact complex space). Then

(i) The cohomology class of a closed positive $(1,1)$-current $\{T\}$ is nef if and only if the restriction $\{T\}_{\mid Z}$ is nef for every irreducible component $Z$ in any of the Lelong sublevel sets $E_{c}(T)$.

(ii) The cohomology class of a Kähler current $\{T\}$ is a Kähler class (i.e. the class of a smooth Kähler form) if and only if the restriction $\{T\}_{\mid Z}$ is a Kähler class for every irreducible component $Z$ in any of the Lelong sublevel sets $E_{c}(T)$.

The proof of Proposition 3.4 is not extremely hard if we take for granted the fact that Kähler currents can be approximated by Kähler currents with logarithmic poles, a fact which was proved in Demailly [14] (see also Theorem 2.10 below). The main point then consists in an induction on dimension and a standard gluing procedure : if $T=\alpha+\frac{i}{\pi} \partial \bar{\partial} \varphi$ where $\varphi$ is smooth on $X \backslash Z$ and has $-\infty$ poles along $Z$, then we can remove the poles of $\varphi$ by replacing $\varphi$ with $\max (\varphi, \psi-C)$, provided $\psi$ is smooth and defined near $Z$ and $C$ is a large constant.

The next (and more substantial step) consists of the following result which is reminiscent of the Grauert-Riemenschneider conjecture (Siu [57], Demailly [11]).

Theorem 3.5. (Demailly-Păun [18]) Let X be a compact Kähler manifold and let $\{\alpha\}$ be a nef class (i.e. $\{\alpha\} \in \overline{\mathcal{K}})$. Assume that $\int_{X} \alpha^{n}>0$. Then $\{\alpha\}$ contains a Kähler current $T$, in other words $\{\alpha\} \in \mathcal{E}^{\circ}$.

Proof. The basic argument is to prove that for every irreducible analytic set $Y \subset X$ of codimension $p$, the class $\{\alpha\}^{p}$ contains a closed positive $(p, p)$-current $\Theta$ such that $\Theta \geq \delta[Y]$ for some $\delta>0$. We check this by observing that $\alpha+\varepsilon \omega$ is a Kähler class, hence by the Calabi-Yau theorem Yau [69] the Monge-Ampère equation

$$
\left(\alpha+\varepsilon \omega+i \partial \bar{\partial} \varphi_{\varepsilon}\right)^{n}=f_{\varepsilon}
$$

can be solved with an arbitrary right-hand side $f_{\varepsilon}>0$ such that

$$
\int_{X} f_{\varepsilon}=C_{\varepsilon}=\int_{X}(\alpha+\varepsilon \omega)^{n}
$$


However, by our assumption that $\int_{X} \alpha^{n}>0$, the constant $C_{\varepsilon}$ is bounded away from 0 . We use this fact in order to concentrate a fixed amount of volume of the volume form $f_{\varepsilon}$ in an $\varepsilon$-tubular neighborhood of $Y$. We then show that the sequence of $(p, p)$-forms $\left(\alpha+\varepsilon \omega+i \partial \bar{\partial} \varphi_{\varepsilon}\right)^{p}$ converges weakly to the desired current $\Theta$ (this part relies heavily on the theory of currents). The second and final part uses a "diagonal trick": apply the result just proved to

$$
\widetilde{X}=X \times X, \quad \widetilde{Y}=\operatorname{diagonal} \subset \widetilde{X}, \quad \widetilde{\alpha}=\operatorname{pr}_{1}^{*} \alpha+\operatorname{pr}_{2}^{*} \alpha .
$$

It is then clear that $\widetilde{\alpha}$ is nef on $\widetilde{X}$ and that $\int_{\widetilde{X}}(\widetilde{\alpha})^{2 n}>0$. It follows by the above that the class $\{\widetilde{\alpha}\}^{n}$ contains a Kähler current $\Theta$ such that $\Theta \geq \delta[\widetilde{Y}]$ for some $\delta>0$. Therefore the push-forward

$$
T:=\left(\operatorname{pr}_{1}\right)_{*}\left(\Theta \wedge \operatorname{pr}_{2}^{*} \omega\right)
$$

is numerically equivalent to a multiple of $\alpha$ and dominates $\delta \omega$, and we see that $T$ is a Kähler current.

End of Proof of Theorem 3.1. Clearly the open cone $\mathcal{K}$ is contained in $\mathcal{P}$, hence in order to show that $\mathcal{K}$ is one of the connected components of $\mathcal{P}$, we need only show that $\mathcal{K}$ is closed in $\mathcal{P}$, i.e. that $\overline{\mathcal{K}} \cap \mathcal{P} \subset \mathcal{K}$. Pick a class $\{\alpha\} \in \overline{\mathcal{K}} \cap \mathcal{P}$. In particular $\{\alpha\}$ is nef and satisfies $\int_{X} \alpha^{n}>0$. By Theorem 3.5 we conclude that $\{\alpha\}$ contains a Kähler current $T$. However, an induction on dimension using the assumption $\int_{Y} \alpha^{p}$ for all analytic subsets $Y$ (we also use resolution of singularities for $Y$ at this step) shows that the restriction $\{\alpha\}_{\mid Y}$ is the class of a Kähler current on $Y$. We conclude that $\{\alpha\}$ is a Kähler class by 3.4 (ii), therefore $\{\alpha\} \in \mathcal{K}$, as desired.

The projective case 3.2 is a consequence of the following variant of Theorem 3.1.

Corollary 3.6. Let $X$ be a compact Kähler manifold. A $(1,1)$ cohomology class $\{\alpha\}$ on $X$ is Kähler if and only if there exists a Kähler metric $\omega$ on $X$ such that $\int_{Y} \alpha^{k} \wedge \omega^{p-k}>0$ for all irreducible analytic sets $Y$ and all $k=1,2, \ldots, p=\operatorname{dim} Y$.

Proof. The assumption clearly implies that

$$
\int_{Y}(\alpha+t \omega)^{p}>0
$$

for all $t \in \mathbb{R}_{+}$, hence the half-line $\alpha+\left(\mathbb{R}_{+}\right) \omega$ is entirely contained in the cone $\mathcal{P}$ of numerically positive classes. Since $\alpha+t_{0} \omega$ is Kähler for $t_{0}$ large, we conclude that the half-line in entirely contained in the connected component $\mathcal{K}$, and therefore $\alpha \in \mathcal{K}$.

In the projective case, we can take $\omega=c_{1}(H)$ for a given very ample divisor $H$, and the condition $\int_{Y} \alpha^{k} \wedge \omega^{p-k}>0$ is equivalent to $\int_{Y \cap H_{1} \cap \ldots \cap H_{p-k}} \alpha^{k}>0$ for a suitable complete intersection $Y \cap H_{1} \cap \ldots \cap H_{p-k}, H_{j} \in|H|$. This shows that algebraic cycles are sufficient to test the Kähler property, and the special case 3.2 follows. On the other hand, we can pass to the limit in 3.6 by replacing $\alpha$ by $\alpha+\varepsilon \omega$, and in this way we get also a characterization of nef classes. 
Corollary 3.7. Let $X$ be a compact Kähler manifold. A $(1,1)$ cohomology class $\{\alpha\}$ on $X$ is nef if and only if there exists a Kähler metric $\omega$ on $X$ such that $\int_{Y} \alpha^{k} \wedge \omega^{p-k} \geq 0$ for all irreducible analytic sets $Y$ and all $k=1,2, \ldots, p=\operatorname{dim} Y$.

By a formal convexity argument, one can derive from 3.6 or 3.7 the following interesting consequence about the dual of the cone $\mathcal{K}$.

Theorem 3.8. Let $X$ be a compact Kähler manifold. A $(1,1)$ cohomology class $\{\alpha\}$ on $X$ is nef if and only for every irreducible analytic set $Y$ in $X, p=\operatorname{dim} X$ and every Kähler metric $\omega$ on $X$ we have $\int_{Y} \alpha \wedge \omega^{p-1} \geq 0$. In other words, the dual of the nef cone $\overline{\mathcal{K}}$ is the closed convex cone in $H_{\mathbb{R}}^{n-1, n-1}(X)$ generated by cohomology classes of currents of the form $[Y] \wedge \omega^{p-1}$ in $H^{n-1, n-1}(X, \mathbb{R})$, where $Y$ runs over the collection of irreducible analytic subsets of $X$ and $\{\omega\}$ over the set of Kähler classes of $X$.

\section{Deformations of compact Kähler manifolds}

If $S$ is an analytic space, recall that a deformation of compact complex manifolds is a proper holomorphic map $\pi: \mathcal{X} \rightarrow S$ such that the fibers are smooth and such that $\mathcal{X}$ is locally the product of the base by a neighborhood of any point in any fiber (with $\pi$ being the first projection of such a local decomposition). For any $t \in S$, we denote by $X_{t}=\pi^{-1}(t)$ the fiber over $t$.

Since compact Kähler manifolds share many common features with projective algebraic manifolds - e.g. good Hodge theoretic properties - rather strong properties are expected for their deformation theory. Kodaira showed in the 60's that every Kähler surface $X$ is a limit by deformation of algebraic surfaces, namely there exists a deformation $\mathcal{X} \rightarrow S$ such that $X=X_{t_{0}}$ for some $t_{0}$, and $X_{t_{m}}$ is projective algebraic for a sequence $t_{m} \rightarrow t_{0}$. It was therefore a natural - and long-standing question whether a similar property holds in higher dimensions. C. Voisin showed in a series of recent papers that the general answer is negative, and in fact there exist rigid non projective compact Kähler manifolds.

Theorem 4.1. (recent results by C. Voisin [67], [68])

(i) In any dimension $\geq 4$, there exist compact Kähler manifolds which do not have the homotopy type (or even the homology ring) of a complex projective manifold ([67]).

(ii) In any dimension $\geq 8$, there exist compact Kähler manifolds $X$ such that no compact bimeromorphic model $X^{\prime}$ of $X$ has the homotopy type of a complex projective manifold ([68]).

The example in (i) is obtained by selecting a complex torus $T$ of dimension $\geq 2$ possessing a linear endomorphism $\varphi$ which has non real eigenvalues (pairwise distinct and non conjugate). Then $X$ is obtained by blowing-up the finite set of pairwise intersection points of of the four subsets $T \times\{0\},\{0\} \times T, \Delta=$ diagonal, $G_{\varphi}=$ graph of $\varphi$, and then their strict transforms in the first stage blow-up. By 
using rather elementary considerations of Hodge theory, this provides an example of a rigid Kähler variety which does not have the homotopy type of a projective variety. The example in (ii) is obtained via the Poincaré bundle on $T \times \hat{T}$; we refer to [67] and [68] for details.

Another fundamental fact proved by Kodaira and Spencer [37] is the observation that the Kähler property is open with respect to deformation: if $X_{t_{0}}$ is Kähler for some $t_{0} \in S$, then the nearby fibers $X_{t}$ (for $t$ in a metric topology neighborhood of $t_{0}$ in $S$ ) is also Kähler. The proof consists in showing that the desired Kähler metrics are solutions of a suitably chosen 4-th order elliptic differential operator for which there is no jump of the kernel at $t_{0}$. However, the numerous known examples leave hopes for a much stronger openness property.

Conjecture 4.2. Let $\mathcal{X} \rightarrow S$ be a deformation with irreducible base space $S$ such that some fiber $X_{t_{0}}$ is Kähler. Then there should exist a finite (or possibly countable) union of analytic strata $S_{\nu} \subset S, S_{\nu} \neq S$, such that

(i) $X_{t}$ is Kähler for $t \in S \backslash \bigcup S_{\nu}$.

(ii) $X_{t}$ is bimeromorphic to a Kähler manifold for $t \in \bigcup S_{\nu}$.

A crucial step in analyzing the conjecture is to describe the behaviour of the Kähler cone of $X_{t}$ as $t$ approaches the "bad strata". This question is now fully understood thanks to the following result which is a direct corollary of our characterization of the Kähler cone (Theorem 3.1). As a consequence, a "collapse" of the Kähler cone could only come from a degeneration of the Hodge decomposition, the behaviour of which is complex analytic thanks to the Frölicher spectral sequence.

Theorem 4.3. (Demailly-Păun [18]) Let $\pi: \mathcal{X} \rightarrow S$ be a deformation of compact Kähler manifolds over an irreducible base $S$. Then there exists a countable union $S^{\prime}=\bigcup S_{\nu}$ of analytic subsets $S_{\nu} \subsetneq S$, such that the Kähler cones $\mathcal{K}_{t} \subset H^{1,1}\left(X_{t}, \mathbb{C}\right)$ of the fibers $X_{t}=\pi^{-1}(t)$ are invariant over $S \backslash S^{\prime}$ under parallel transport with respect to the $(1,1)$-projection $\nabla^{1,1}$ of the Gauss-Manin connection $\nabla$ in the decomposition of

$$
\nabla=\left(\begin{array}{ccc}
\nabla^{2,0} & * & 0 \\
* & \nabla^{1,1} & * \\
0 & * & \nabla^{0,2}
\end{array}\right)
$$

on the Hodge bundle $H^{2}=H^{2,0} \oplus H^{1,1} \oplus H^{0,2}$.

Sketch of Proof. The result is local on the base, hence we may assume that $S$ is contractible. Then the family is differentiably trivial, the Hodge bundle $t \mapsto$ $H^{2}\left(X_{t}, \mathbb{C}\right)$ is the trivial bundle and $t \mapsto H^{2}\left(X_{t}, \mathbb{Z}\right)$ is a trivial lattice. We use the existence of a relative cycle space $C^{p}(\mathcal{X} / S) \subset C^{p}(\mathcal{X})$ which consists of all cycles contained in the fibres of $\pi: X \rightarrow S$. It is equipped with a canonical holomorphic projection

$$
\pi_{p}: C^{p}(\mathcal{X} / S) \rightarrow S
$$

We then define the $S_{\nu}$ 's to be the images in $S$ of those connected components of $C^{p}(\mathcal{X} / S)$ which do not project onto $S$. By the fact that the projection is proper 
on each component, we infer that $S_{\nu}$ is an analytic subset of $S$. The definition of the $S_{\nu}$ 's implies that the cohomology classes induced by the analytic cycles $\{[Z]\}$, $Z \subset X_{t}$, remain exactly the same for all $t \in S \backslash S^{\prime}$. This result implies in its turn that the conditions defining the numerically positive cones $\mathcal{P}_{t}$ remain the same, except for the fact that the spaces $H^{1,1}\left(X_{t}, \mathbb{R}\right) \subset H^{2}\left(X_{t}, \mathbb{R}\right)$ vary along with the Hodge decomposition. At this point, a standard calculation implies that the $\mathcal{P}_{t}$ are invariant by parallel transport under $\nabla^{1,1}$. Moreover, the connected component $\mathcal{K}_{t} \subset \mathcal{P}_{t}$ cannot jump from one component to the other thanks to the already metionned results by Kodaira-Spencer [37]. This concludes the proof.

Theorem 4.3 was essentially already known in the cases of complex surfaces (i.e. in dimension 2), thanks to the work of N. Buchdahl [6], [7] and A. Lamari [38], [39].

Shortly after the original [18] manuscript appeared in April 2001, Daniel Huybrechts [27] informed us Theorem 3.1 can be used to calculate the Kähler cone of a very general hyperkähler manifold: the Kähler cone is then equal to a suitable connected component of the positive cone defined by the Beauville-Bogomolov quadratic form. In the case of an arbitrary hyperkähler manifold, S.Boucksom [Bou02] later showed that a $(1,1)$ class $\{\alpha\}$ is Kähler if and only if it lies in the positive part of the Beauville-Bogomolov quadratic cone and moreover $\int_{C} \alpha>0$ for all rational curves $C \subset X$ (see also Huybrechts [26]).

\section{Positive cones in $H^{n-1, n-1}(X)$ and Serre duality}

5.1. Basic definitions. In a way which will be shown to be dual to the case of divisors and positive $(1,1)$-currents, we consider in $H_{\mathbb{R}}^{n-1, n-1}(X)$ the cone $\mathcal{N}$ generated by classes of positive currents $T$ of type $(n-1, n-1)$ (i.e., of bidimension $(1,1))$. In the projective case, we also consider the intersection of $\mathcal{N}$ with the space $N_{1}(X)$ generated by integral $(n-1, n-1)$-classes (by the hard Lefschetz theorem, $N_{1}(X)$ is just the dual of $\left.N S_{\mathbb{R}}(X)\right)$.

Definition 5.1. Let $X$ be a compact Kähler manifold.

(i) We define $\mathcal{N}$ to be the (closed) convex cone in $H_{\mathbb{R}}^{n-1, n-1}(X)$ generated by classes of positive currents $T$ of type $(n-1, n-1)$ (i.e., of bidimension $(1,1))$.

(ii) We define the cone $\mathcal{M} \subset H_{\mathbb{R}}^{n-1, n-1}(X)$ of "movable classes" to be the closure of the convex cone generated by classes of currents of the form

$$
\mu_{\star}\left(\widetilde{\omega}_{1} \wedge \ldots \wedge \widetilde{\omega}_{n-1}\right)
$$

where $\mu: \widetilde{X} \rightarrow X$ is an arbitrary modification (one could just restrict oneself to compositions of blow-ups with smooth centers), and the $\widetilde{\omega}_{j}$ are Kähler forms on $\tilde{X}$. Clearly $\mathcal{M} \subset \mathcal{N}$. 
(iii) Correspondingly, we introduce the intersections

$$
\mathcal{N}_{\mathrm{NS}}=\mathcal{N} \cap N_{1}(X), \quad \mathcal{M}_{\mathrm{NS}}=\mathcal{M} \cap N_{1}(X),
$$

in the space generated by integral bidimension $(1,1)$-classes

$$
N_{1}(X):=\left(H_{\mathbb{R}}^{n-1, n-1}(X) \cap H^{2 n-2}(X, \mathbb{Z}) /\{\text { torsion }\}\right) \otimes_{\mathbb{Z}} \mathbb{R} .
$$

(iv) If $X$ is projective, we define $\mathrm{NE}(X)$ to be the convex cone generated by all effective curves. Clearly $\overline{\mathrm{NE}(X)} \subset \mathcal{N}_{\mathrm{NS}}$.

(v) If $X$ is projective, we say that $C$ is a "strongly movable" curve if

$$
C=\mu_{\star}\left(\widetilde{A}_{1} \cap \ldots \cap \widetilde{A}_{n-1}\right)
$$

for suitable very ample divisors $\widetilde{A}_{j}$ on $\widetilde{X}$, where $\mu: \widetilde{X} \rightarrow X$ is a modification. We let $\operatorname{SME}(X)$ be the convex cone generated by all strongly movable (effective) curves. Clearly $\overline{\operatorname{SME}(X)} \subset \mathcal{M}_{\mathrm{NS}}$.

(vi) We say that $C$ is a movable curve if $C=C_{t_{0}}$ is a member of an analytic family $\left(C_{t}\right)_{t \in S}$ such that $\bigcup_{t \in S} C_{t}=X$ and, as such, is a reduced irreducible 1-cycle. We let $\operatorname{ME}(X)$ to be the convex cone generated by all movable (effective) curves.

The upshot of this definition lies in the following easy observation.

Proposition 5.2. Let $X$ be a compact Kähler manifold. Consider the Poincaré duality pairing

$$
H^{1,1}(X, \mathbb{R}) \times H_{\mathbb{R}}^{n-1, n-1}(X) \longrightarrow \mathbb{R}, \quad(\alpha, \beta) \longmapsto \int_{X} \alpha \wedge \beta .
$$

Then the duality pairing takes nonnegative values

(i) for all pairs $(\alpha, \beta) \in \overline{\mathcal{K}} \times \mathcal{N}$;

(ii) for all pairs $(\alpha, \beta) \in \mathcal{E} \times \mathcal{M}$.

(iii) for all pairs $(\alpha, \beta)$ where $\alpha \in \mathcal{E}$ and $\beta=\left[C_{t}\right] \in \operatorname{ME}(X)$ is the class of a movable curve.

Proof. (i) is obvious. In order to prove (ii), we may assume that $\beta=\mu_{\star}\left(\widetilde{\omega}_{1} \wedge \ldots \wedge\right.$ $\left.\widetilde{\omega}_{n-1}\right)$ for some modification $\mu: \widetilde{X} \rightarrow X$, where $\alpha=\{T\}$ is the class of a positive $(1,1)$-current on $X$ and $\widetilde{\omega}_{j}$ are Kähler forms on $\widetilde{X}$. Then

$$
\int_{X} \alpha \wedge \beta=\int_{X} T \wedge \mu_{\star}\left(\widetilde{\omega}_{1} \wedge \ldots \wedge \widetilde{\omega}_{n-1}\right)=\int_{X} \mu^{*} T \wedge \widetilde{\omega}_{1} \wedge \ldots \wedge \widetilde{\omega}_{n-1} \geq 0 .
$$

Here, we have used the fact that a closed positive $(1,1)$-current $T$ always has a pull-back $\mu^{\star} T$, which follows from the fact that if $T=i \partial \bar{\partial} \varphi$ locally for some 
plurisubharmonic function in $X$, we can set $\mu^{\star} T=i \partial \bar{\partial}(\varphi \circ \mu)$. For (iii), we suppose $\alpha=\{T\}$ and $\beta=\left\{\left[C_{t}\right]\right\}$. Then we take an open covering $\left(U_{j}\right)$ on $X$ such that $T=i \partial \bar{\partial} \varphi_{j}$ with suitable plurisubharmonic functions $\varphi_{j}$ on $U_{j}$. If we select a smooth partition of unity $\sum \theta_{j}=1$ subordinate to $\left(U_{j}\right)$, we then get

$$
\int_{X} \alpha \wedge \beta=\int_{C_{t}} T_{\mid C_{t}}=\sum_{j} \int_{C_{t} \cap U_{j}} \theta_{j} i \partial \bar{\partial} \varphi_{j \mid C_{t}} \geq 0 .
$$

For this to make sense, it should be noticed that $T_{\mid C_{t}}$ is a well defined closed positive $(1,1)$-current (i.e. measure) on $C_{t}$ for almost every $t \in S$, in the sense of Lebesgue measure. This is true only because $\left(C_{t}\right)$ covers $X$, thus $\varphi_{j \mid C_{t}}$ is not identically $-\infty$ for almost every $t \in S$. The equality in the last formula is then shown by a regularization argument for $T$, writing $T=\lim T_{k}$ with $T_{k}=\alpha+i \partial \bar{\partial} \psi_{k}$ and a decreasing sequence of smooth almost plurisubharmonic potentials $\psi_{k} \downarrow \psi$ such that the Levi forms have a uniform lower bound $i \partial \bar{\partial} \psi_{k} \geq-C \omega$ (such a sequence exists by Demailly [14]). Then, writing $\alpha=i \partial \bar{\partial} v_{j}$ for some smooth potential $v_{j}$ on $U_{j}$, we have $T=i \partial \bar{\partial} \varphi_{j}$ on $U_{j}$ with $\varphi_{j}=v_{j}+\psi$, and this is the decreasing limit of the smooth approximations $\varphi_{j, k}=v_{j}+\psi_{k}$ on $U_{j}$. Hence $T_{k \mid C_{t}} \rightarrow T_{\mid C_{t}}$ for the weak topology of measures on $C_{t}$.

If $\mathcal{C}$ is a convex cone in a finite dimensional vector space $E$, we denote by $\mathcal{C}^{\vee}$ the dual cone, i.e. the set of linear forms $u \in E^{\star}$ which take nonnegative values on all elements of $\mathcal{C}$. By the Hahn-Banach theorem, we always have $\mathcal{C}^{\vee \vee}=\overline{\mathcal{C}}$. Proposition 5.2 leads to the natural question whether the cones $(\mathcal{K}, \mathcal{N})$ and $(\mathcal{E}, \mathcal{M})$ are dual under Poincaré duality, according to the following schematic picture.

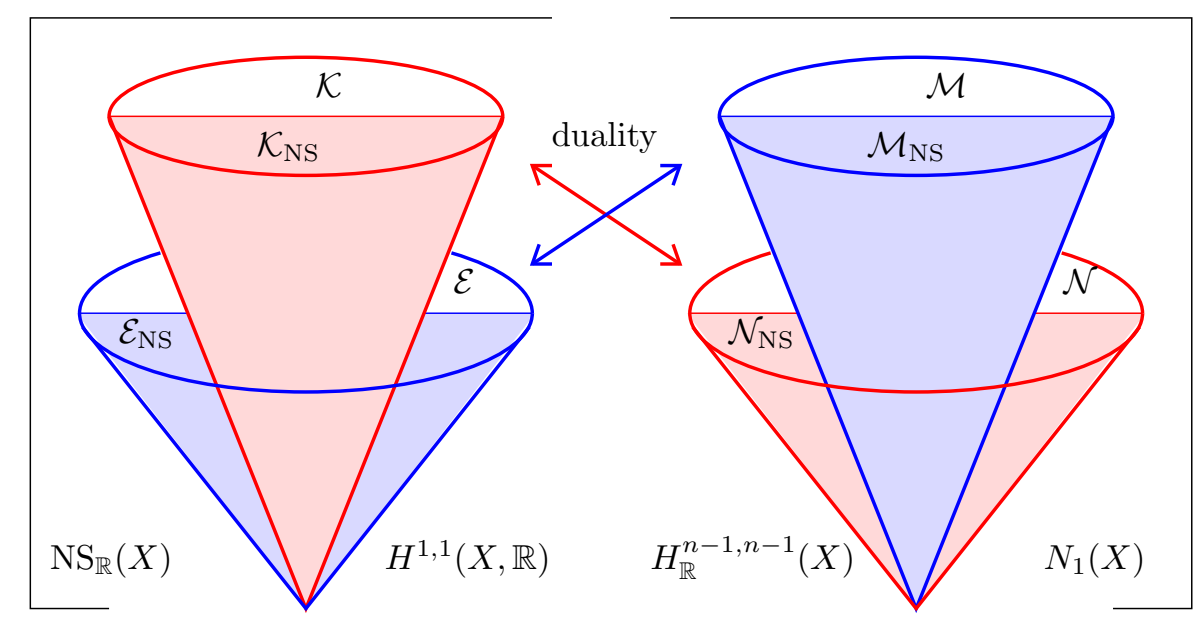

It is indeed well-known that the cone $\overline{\mathcal{K}_{\mathrm{NS}}}$ of nef divisors is dual to the cone $\mathcal{N}_{\mathrm{NS}}$ of effective curves if $X$ is projective. The transcendental version $\mathcal{K}=\mathcal{N}^{\vee}$ also follows from our Theorem 3.8. 
Theorem 5.3. (Demailly-Păun) If $X$ is Kähler, then the cones $\overline{\mathcal{K}} \subset H^{1,1}(X, \mathbb{R})$ and $\mathcal{N} \subset H_{\mathbb{R}}^{n-1, n-1}(X)$ are dual by Poincaré duality, and $\mathcal{N}$ is the closed convex cone generated by classes $[Y] \wedge \omega^{p-1}$ where $Y \subset X$ ranges over p-dimensional analytic subsets, $p=1,2, \ldots, n$, and $\omega$ ranges over Kähler forms.

Proof. Indeed, Prop. 5.2 shows that the dual cone $\mathcal{K}^{\vee}$ contains $\mathcal{N}$ which itself contains the cone $\mathcal{N}^{\prime}$ of all classes of the form $\left\{[Y] \wedge \omega^{p-1}\right\}$. The main result of Demailly-Păun [18] conversely shows that the dual of $\left(\mathcal{N}^{\prime}\right)^{\vee}$ is equal to $\overline{\mathcal{K}}$, so we must have

$$
\mathcal{K}^{\vee}=\overline{\mathcal{N}^{\prime}}=\mathcal{N} .
$$

The other duality statement $\mathcal{E}=\mathcal{M}^{\vee}$ will be investigated in the next sections.

5.2. Concept of volume and movable intersections. We start with the very important concept of volume.

Definition 5.4. We define the volume, or movable self-intersection of a big class $\alpha \in \mathcal{E}^{\circ}$ to be

$$
\operatorname{Vol}(\alpha)=\sup _{T \in \alpha} \int_{\widetilde{X}} \beta^{n}>0
$$

where the supremum is taken over all Kähler currents $T \in \alpha$ with logarithmic poles, and $\mu^{\star} T=[E]+\beta$ with respect to some modification $\mu: \widetilde{X} \rightarrow X$.

By Fujita [21] and Demailly-Ein-Lazarsfeld [17], if $L$ is a big line bundle, we have

$$
\operatorname{Vol}\left(c_{1}(L)\right)=\lim _{m \rightarrow+\infty} D_{m}^{n}=\lim _{m \rightarrow+\infty} \frac{n !}{m^{n}} h^{0}(X, m L),
$$

and in these terms, we get the following statement.

Proposition 5.5. Let $L$ be a big line bundle on the projective manifold $X$. Let $\varepsilon>0$. Then there exists a modification $\mu: X_{\varepsilon} \rightarrow X$ and a decomposition $\mu^{*}(L)=$ $E+\beta$ with $E$ an effective $\mathbb{Q}$-divisor and $\beta$ a big and nef $\mathbb{Q}$-divisor such that

$$
\operatorname{Vol}(L)-\varepsilon \leq \operatorname{Vol}(\beta) \leq \operatorname{Vol}(L)
$$

It is very useful to observe that the supremum in Definition 5.4 is actually achieved by a collection of currents whose singularities satisfy a filtering property. Namely, if $T_{1}=\alpha+i \partial \bar{\partial} \varphi_{1}$ and $T_{2}=\alpha+i \partial \bar{\partial} \varphi_{2}$ are two Kähler currents with logarithmic poles in the class of $\alpha$, then

$$
T=\alpha+i \partial \bar{\partial} \varphi, \quad \varphi=\max \left(\varphi_{1}, \varphi_{2}\right)
$$

is again a Kähler current with weaker singularities than $T_{1}$ and $T_{2}$. One could define as well

$$
T=\alpha+i \partial \bar{\partial} \varphi, \quad \varphi=\frac{1}{2 m} \log \left(e^{2 m \varphi_{1}}+e^{2 m \varphi_{2}}\right),
$$

where $m=\operatorname{lcm}\left(m_{1}, m_{2}\right)$ is the lowest common multiple of the denominators occuring in $T_{1}, T_{2}$. Now, take a simultaneous log-resolution $\mu_{m}: \widetilde{X}_{m} \rightarrow X$ for 
which the singularities of $T_{1}$ and $T_{2}$ are resolved as $\mathbb{Q}$-divisors $E_{1}$ and $E_{2}$. Then clearly the associated divisor in the decomposition $\mu_{m}^{\star} T=[E]+\beta$ is given by $E=\min \left(E_{1}, E_{2}\right)$.

Theorem 5.6. (Boucksom [4]) Let $X$ be a compact Kähler manifold. We denote here by $H_{\geq 0}^{k, k}(X)$ the cone of cohomology classes of type $(k, k)$ which have nonnegative intersection with all closed semi-positive smooth forms of bidegree $(n-$ $k, n-k)$.

(i) For each $k=1, \ldots, n$, there exists a canonical "movable intersection product"

$$
\mathcal{E} \times \cdots \times \mathcal{E} \rightarrow H_{\geq 0}^{k, k}(X), \quad\left(\alpha_{1}, \ldots, \alpha_{k}\right) \mapsto\left\langle\alpha_{1} \cdot \alpha_{2} \cdots \alpha_{k-1} \cdot \alpha_{k}\right\rangle
$$

such that $\operatorname{Vol}(\alpha)=\left\langle\alpha^{n}\right\rangle$ whenever $\alpha$ is a big class.

(ii) The product is increasing, homogeneous of degree 1 and superadditive in each argument, i.e.

$$
\left\langle\alpha_{1} \cdots\left(\alpha_{j}^{\prime}+\alpha_{j}^{\prime \prime}\right) \cdots \alpha_{k}\right\rangle \geq\left\langle\alpha_{1} \cdots \alpha_{j}^{\prime} \cdots \alpha_{k}\right\rangle+\left\langle\alpha_{1} \cdots \alpha_{j}^{\prime \prime} \cdots \alpha_{k}\right\rangle .
$$

It coincides with the ordinary intersection product when the $\alpha_{j} \in \overline{\mathcal{K}}$ are nef classes.

(iii) The movable intersection product satisfies the Teissier-Hovanskii inequalities

$$
\left\langle\alpha_{1} \cdot \alpha_{2} \cdots \alpha_{n}\right\rangle \geq\left(\left\langle\alpha_{1}^{n}\right\rangle\right)^{1 / n} \ldots\left(\left\langle\alpha_{n}^{n}\right\rangle\right)^{1 / n} \quad\left(\text { with }\left\langle\alpha_{j}^{n}\right\rangle=\operatorname{Vol}\left(\alpha_{j}\right)\right) .
$$

(iv) For $k=1$, the above "product" reduces to a (non linear) projection operator

$$
\mathcal{E} \rightarrow \mathcal{E}_{1}, \quad \alpha \rightarrow\langle\alpha\rangle
$$

onto a certain convex subcone $\mathcal{E}_{1}$ of $\mathcal{E}$ such that $\overline{\mathcal{K}} \subset \mathcal{E}_{1} \subset \mathcal{E}$. Moreover, there is a "divisorial Zariski decomposition"

$$
\alpha=\{N(\alpha)\}+\langle\alpha\rangle
$$

where $N(\alpha)$ is a uniquely defined effective divisor which is called the "negative divisorial part" of $\alpha$. The map $\alpha \mapsto N(\alpha)$ is homogeneous and subadditive, and $N(\alpha)=0$ if and only if $\alpha \in \mathcal{E}_{1}$.

(v) The components of $N(\alpha)$ always consist of divisors whose cohomology classes are linearly independent, thus $N(\alpha)$ has at most $\rho=\operatorname{rank}_{\mathbb{Z}} \mathrm{NS}(X)$ components.

Proof. We refer to S. Boucksom's thesis [4] for details. Boucksom's treatment also covers the case of compact non Kähler manifolds, so it is fairly general. We only give a very rough construction of the movable intersection product.

First assume that all classes $\alpha_{j}$ are big, i.e. $\alpha_{j} \in \mathcal{E}^{\circ}$. We select Kähler currents $T_{j, m} \in \alpha_{j}$ with logarithmic poles and their approximate Zariski decompositions as 
in Theorem 2.10. We can then find a simultaneous log-resolution $\mu_{m}: \widetilde{X}_{m} \rightarrow X$ such that

$$
\mu^{\star} T_{j, m}=\left[E_{j}, m\right]+\beta_{j, m}
$$

We consider the direct image current $\mu_{m \star}\left(\beta_{1, m} \wedge \ldots \wedge \beta_{k, m}\right)$ (which is a closed positive current of bidegree $(k, k)$ on $X)$. It turns out by rather elementary monotonicity arguments based on the filtering property 5.2 that one can can extract a weakly convergent limit

$$
\left\langle\alpha_{1} \cdot \alpha_{2} \cdots \alpha_{k}\right\rangle=\lim _{m \rightarrow+\infty}\left\{\left(\mu_{m}\right)_{\star}\left(\beta_{1, m} \wedge \beta_{2, m} \wedge \ldots \wedge \beta_{k, m}\right)\right\}
$$

and that the corresponding cohomology class in $H^{k, k}(X)$ is uniquely defined. Now, the intersection product can be extended to the full closed cone $\mathcal{E}$ by monotonicity again, namely by setting

$$
\left\langle\alpha_{1} \cdot \alpha_{2} \cdots \alpha_{k}\right\rangle=\lim _{\delta \downarrow 0} \downarrow\left\langle\left(\alpha_{1}+\delta \omega\right) \cdot\left(\alpha_{2}+\delta \omega\right) \cdots\left(\alpha_{k}+\delta \omega\right)\right\rangle
$$

for arbitrary classes $\alpha_{j} \in \mathcal{E}$.

Definition 5.7. For a class $\alpha \in H^{1,1}(X, \mathbb{R})$, we define the numerical dimension $\nu(\alpha)$ to be $\nu(\alpha)=-\infty$ if $\alpha$ is not pseudo-effective, and

$$
\nu(\alpha)=\max \left\{p \in \mathbb{N} ;\left\langle\alpha^{p}\right\rangle \neq 0\right\}, \quad \nu(\alpha) \in\{0,1, \ldots, n\}
$$

if $\alpha$ is pseudo-effective.

By the results of Demailly-Peternell [18], a class is big $\left(\alpha \in \mathcal{E}^{\circ}\right)$ if and only if $\nu(\alpha)=n$. Classes of numerical dimension 0 can be described much more precisely, again following Boucksom [4].

Theorem 5.8. Let $X$ be a compact Kähler manifold. Then the subset $\mathcal{D}_{0}$ of irreducible divisors $D$ in $X$ such that $\nu(D)=0$ is countable, and these divisors are rigid as well as their multiples. If $\alpha \in \mathcal{E}$ is a pseudo-effective class of numerical dimension 0 , then $\alpha$ is numerically equivalent to an effective $\mathbb{R}$-divisor $D=\sum_{j \in J} \lambda_{j} D_{j}$, for some finite subset $\left(D_{j}\right)_{j \in J} \subset \mathcal{D}_{0}$ such that the cohomology classes $\left\{D_{j}\right\}$ are linearly independent and some $\lambda_{j}>0$. If such a linear combination is of numerical dimension 0 , then so is any other linear combination of the same divisors.

Using the Iitaka fibration, it is immediate to see that $\kappa(X) \leq \nu(X)$ always holds true, and from the currently known examples a natural expectation would be

Conjecture 5.9. ("generalized abundance conjecture") For an arbitrary compact Kähler manifold $X$, the Kodaira dimension should be equal to the numerical dimension :

$$
\kappa(X)=\nu(X):=\nu\left(c_{1}\left(K_{X}\right)\right)
$$


This appears to be a fairly strong statement. In fact, it is not difficult to show that the generalized abundance conjecture contains the $C_{n, m}$ conjectures about additivity of Kodaira dimension (since it is not very difficult to show that the numerical dimension is additive with respect to fibrations). A few extreme cases are known.

Theorem 5.10. The generalized abundance conjecture is true at least in the cases $\nu(X)=-\infty, \nu(X)=0, \nu(X)=n$.

Proof. In fact $\nu(X)=-\infty$ means that $K_{X}$ is not pseudo-effective, so no multiple of $K_{X}$ can have sections and thus $\kappa(X)=-\infty$. In case $\nu(X)=n$, we have to show that $K_{X}$ is big $\left(K_{X} \in \mathcal{E}^{\circ}\right)$; this follows from [18] and from the solution of the Grauert-Riemenschneider conjecture in the form proven in Demailly [11]. Remains the case $\nu(X)=0$. Then Theorem 5.8 gives $K_{X} \equiv \sum \lambda_{j} D_{j}$ for some effective divisor with numerically independent components such that $\nu\left(D_{j}\right)=0$. It follows that the $\lambda_{j}$ are rational and therefore

$$
K_{X}=\sum \lambda_{j} D_{j}+F \quad \text { where } \lambda_{j} \in \mathbb{Q}^{+}, \nu\left(D_{j}\right)=0 \text { and } F \in \operatorname{Pic}^{0}(X)
$$

In that case Campana and Peternell [8] have shown that $F$ is a torsion element of $\operatorname{Pic}^{0}(X)$, and so $\kappa(X)=0$.

5.3. The orthogonality estimate. The goal of this section is to show that, in an appropriate sense, approximate Zariski decompositions are almost orthogonal.

Theorem 5.11. Let $X$ be a projective manifold, and let $\alpha=\{T\} \in \mathcal{E}_{\mathrm{NS}}^{\circ}$ be a big class represented by a Kähler current $T$. Consider an approximate Zariski decomposition

$$
\mu_{m}^{\star} T_{m}=\left[E_{m}\right]+\left[D_{m}\right]
$$

Then

$$
\left(D_{m}^{n-1} \cdot E_{m}\right)^{2} \leq 20(C \omega)^{n}\left(\operatorname{Vol}(\alpha)-D_{m}^{n}\right)
$$

where $\omega=c_{1}(H)$ is a Kähler form and $C \geq 0$ is a constant such that $\pm \alpha$ is dominated by $C \omega$ (i.e., $C \omega \pm \alpha$ is nef).

Proof. For every $t \in[0,1]$, we have

$$
\operatorname{Vol}(\alpha)=\operatorname{Vol}\left(E_{m}+D_{m}\right) \geq \operatorname{Vol}\left(t E_{m}+D_{m}\right) .
$$

Now, by our choice of $C$, we can write $E_{m}$ as a difference of two nef divisors

$$
E_{m}=\mu^{\star} \alpha-D_{m}=\mu_{m}^{\star}(\alpha+C \omega)-\left(D_{m}+C \mu_{m}^{\star} \omega\right) .
$$

Lemma 5.12. For all nef $\mathbb{R}$-divisors $A, B$ we have

$$
\operatorname{Vol}(A-B) \geq A^{n}-n A^{n-1} \cdot B
$$

as soon as the right hand side is positive. 
Proof. In case $A$ and $B$ are integral (Cartier) divisors, this is a consequence of the holomorphic Morse inequalities, (Demailly [16], 8.5). If $A$ and $B$ are $\mathbb{Q}$-Cartier, we conclude by the homogeneity of the volume. The general case of $\mathbb{R}$-divisors follows by approximation using the upper semi-continuity of the volume (Boucksom [4], 3.1.26). In fact, we expect Lemma 5.12 to hold true also in the case of transcendental nef cohomology classes - unfortunately the required generalization of Morse inequalities is still missing at this point.

End of proof of Theorem 5.11. In order to exploit the lower bound of the volume, we write

$$
t E_{m}+D_{m}=A-B, \quad A=D_{m}+t \mu_{m}^{\star}(\alpha+C \omega), \quad B=t\left(D_{m}+C \mu_{m}^{\star} \omega\right) .
$$

By our choice of the constant $C$, both $A$ and $B$ are nef. Lemma 5.12 and the binomial formula imply

$$
\begin{aligned}
\operatorname{Vol}\left(t E_{m}+D_{m}\right) & \geq A^{n}-n A^{n-1} \cdot B \\
=D_{m}^{n} & +n t D_{m}^{n-1} \cdot \mu_{m}^{\star}(\alpha+C \omega)+\sum_{k=2}^{n} t^{k}\left(\begin{array}{c}
n \\
k
\end{array}\right) D_{m}^{n-k} \cdot \mu_{m}^{\star}(\alpha+C \omega)^{k} \\
& -n t D_{m}^{n-1} \cdot\left(D_{m}+C \mu_{m}^{\star} \omega\right) \\
& -n t^{2} \sum_{k=1}^{n-1} t^{k-1}\left(\begin{array}{c}
n-1 \\
k
\end{array}\right) D_{m}^{n-1-k} \cdot \mu_{m}^{\star}(\alpha+C \omega)^{k} \cdot\left(D_{m}+C \mu_{m}^{\star} \omega\right) .
\end{aligned}
$$

Now, we use the obvious inequalities

$$
D_{m} \leq \mu_{m}^{\star}(C \omega), \quad \mu_{m}^{\star}(\alpha+C \omega) \leq 2 \mu_{m}^{\star}(C \omega), \quad D_{m}+C \mu_{m}^{\star} \omega \leq 2 \mu_{m}^{\star}(C \omega)
$$

in which all members are nef (and where the inequality $\leq$ means that the difference of classes is pseudo-effective). In this way we get

$$
\operatorname{Vol}\left(t E_{m}+D_{m}\right) \geq D_{m}^{n}+n t D_{m}^{n-1} \cdot E_{m}-n t^{2} \sum_{k=1}^{n-1} 2^{k+1} t^{k-1}\left(\begin{array}{c}
n-1 \\
k
\end{array}\right)(C \omega)^{n}
$$

We will always take $t$ smaller than $1 / 10 n$ so that the last summation is bounded by $4(n-1)(1+1 / 5 n)^{n-2}<4 n e^{1 / 5}<5 n$. This implies

$$
\operatorname{Vol}\left(t E_{m}+D_{m}\right) \geq D_{m}^{n}+n t D_{m}^{n-1} \cdot E_{m}-5 n^{2} t^{2}(C \omega)^{n} .
$$

Now, the choice $t=\frac{1}{10 n}\left(D_{m}^{n-1} \cdot E_{m}\right)\left((C \omega)^{n}\right)^{-1}$ gives by substituting

$$
\frac{1}{20} \frac{\left(D_{m}^{n-1} \cdot E_{m}\right)^{2}}{(C \omega)^{n}} \leq \operatorname{Vol}\left(E_{m}+D_{m}\right)-D_{m}^{n} \leq \operatorname{Vol}(\alpha)-D_{m}^{n}
$$

(and we have indeed $t \leq \frac{1}{10 n}$ ), whence Theorem 5.11. Of course, the constant 20 is certainly not optimal. 
Corollary 5.13. If $\alpha \in \mathcal{E}_{\mathrm{NS}}$, then the divisorial Zariski decomposition $\alpha=N(\alpha)+\langle\alpha\rangle$ is such that $\left\langle\alpha^{n-1}\right\rangle \cdot N(\alpha)=0$.

Proof. By replacing $\alpha$ by $\alpha+\delta c_{1}(H)$, one sees that it is sufficient to consider the case where $\alpha$ is big. Then the orthogonality estimate implies

$\left(\mu_{m}\right)_{\star}\left(D_{m}^{n-1}\right) \cdot\left(\mu_{m}\right)_{\star} E_{m}=D_{m}^{n-1} \cdot\left(\mu_{m}\right)^{\star}\left(\mu_{m}\right)_{\star} E_{m} \leq D_{m}^{n-1} \cdot E_{m} \leq C\left(\operatorname{Vol}(\alpha)-D_{m}^{n}\right)^{1 / 2}$.

Since $\left\langle\alpha^{n-1}\right\rangle=\lim \left(\mu_{m}\right)_{\star}\left(D_{m}^{n-1}\right), N(\alpha)=\lim \left(\mu_{m}\right)_{\star} E_{m}$ and $\lim D_{m}^{n}=\operatorname{Vol}(\alpha)$, we get the desired conclusion in the limit.

5.4. Proof of duality between $\mathcal{E}_{\mathrm{NS}}$ and $\mathcal{M}_{\mathrm{NS}}$. The main point is the following characterization of pseudo-effective classes, proved in [5] (the "only if" part already follows from 5.2 (iii)).

Theorem 5.14. (Boucksom-Demailly-Păun-Peternell [5]) If $X$ is projective, then a class $\alpha \in \mathrm{NS}_{\mathbb{R}}(X)$ is pseudo-effective if (and only if) it is in the dual cone of the cone $\operatorname{SME}(X)$ of strongly movable curves.

In other words, a line bundle $L$ is pseudo-effective if (and only if) $L \cdot C \geq 0$ for all movable curves, i.e., $L \cdot C \geq 0$ for every very generic curve $C$ (not contained in a countable union of algebraic subvarieties). In fact, by definition of $\operatorname{SME}(X)$, it is enough to consider only those curves $C$ which are images of generic complete intersection of very ample divisors on some variety $\widetilde{X}$, under a modification $\mu$ : $\widetilde{X} \rightarrow X$. By a standard blowing-up argument, it also follows that a line bundle $L$ on a normal Moishezon variety is pseudo-effective if and only if $L \cdot C \geq 0$ for every movable curve $C$. The Kähler analogue should be :

Conjecture 5.15. For an arbitrary compact Kähler manifold $X$, the cones $\mathcal{E}$ and $\mathcal{M}$ are dual.
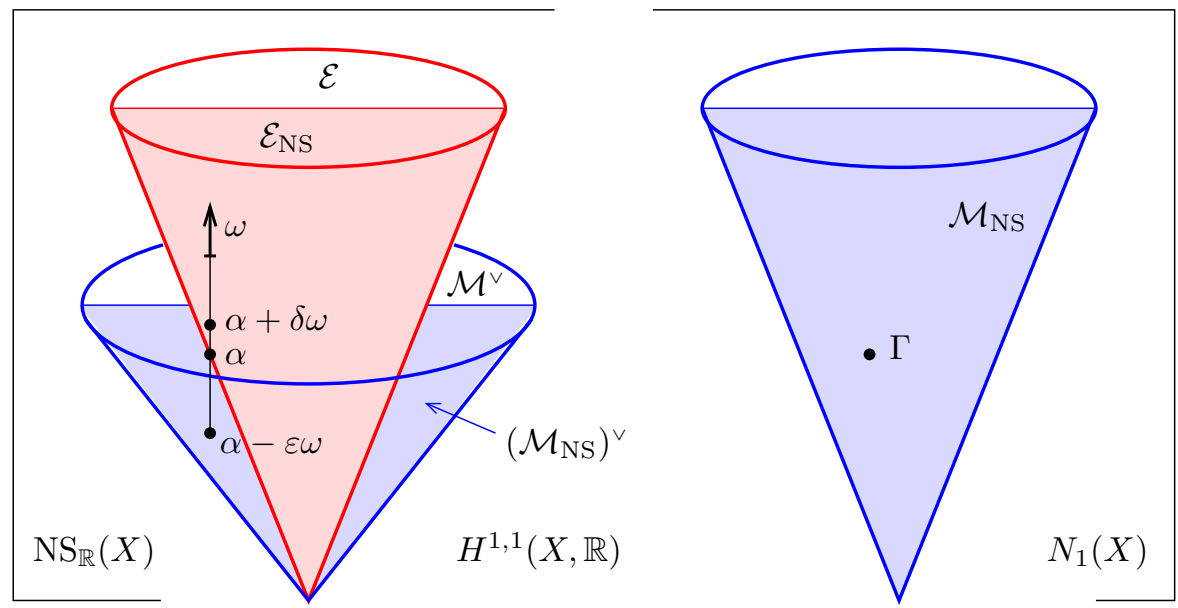
Proof of Theorem 5.14. (see [5]). We want to show that $\mathcal{E}_{\mathrm{NS}}=\operatorname{SME}(X)^{\vee}$. By 5.2 (iii) we have in any case

$$
\mathcal{E}_{\mathrm{NS}} \subset(\operatorname{SME}(X))^{\vee} .
$$

If the inclusion is strict, there is an element $\alpha \in \partial \mathcal{E}_{\mathrm{NS}}$ on the boundary of $\mathcal{E}_{\mathrm{NS}}$ which is in the interior of $\operatorname{SME}(X)^{\vee}$.

Let $\omega=c_{1}(H)$ be an ample class. Since $\alpha \in \partial \mathcal{E}_{\mathrm{NS}}$, the class $\alpha+\delta \omega$ is big for every $\delta>0$, and since $\alpha \in\left((\operatorname{SME}(X))^{\vee}\right)^{\circ}$ we still have $\alpha-\varepsilon \omega \in(\operatorname{SME}(X))^{\vee}$ for $\varepsilon>0$ small. Therefore

$$
\alpha \cdot \Gamma \geq \varepsilon \omega \cdot \Gamma
$$

for every movable curve $\Gamma$. We are going to contradict (5.4). Since $\alpha+\delta \omega$ is big, we have an approximate Zariski decomposition

$$
\mu_{\delta}^{\star}(\alpha+\delta \omega)=E_{\delta}+D_{\delta}
$$

We pick $\Gamma=\left(\mu_{\delta}\right)_{\star}\left(D_{\delta}^{n-1}\right)$. By the Hovanskii-Teissier concavity inequality

$$
\omega \cdot \Gamma \geq\left(\omega^{n}\right)^{1 / n}\left(D_{\delta}^{n}\right)^{(n-1) / n} .
$$

On the other hand

$$
\begin{aligned}
\alpha \cdot \Gamma & =\alpha \cdot\left(\mu_{\delta}\right)_{\star}\left(D_{\delta}^{n-1}\right) \\
& =\mu_{\delta}^{\star} \alpha \cdot D_{\delta}^{n-1} \leq \mu_{\delta}^{\star}(\alpha+\delta \omega) \cdot D_{\delta}^{n-1} \\
& =\left(E_{\delta}+D_{\delta}\right) \cdot D_{\delta}^{n-1}=D_{\delta}^{n}+D_{\delta}^{n-1} \cdot E_{\delta} .
\end{aligned}
$$

By the orthogonality estimate, we find

$$
\begin{aligned}
\frac{\alpha \cdot \Gamma}{\omega \cdot \Gamma} & \leq \frac{D_{\delta}^{n}+\left(20(C \omega)^{n}\left(\operatorname{Vol}(\alpha+\delta \omega)-D_{\delta}^{n}\right)\right)^{1 / 2}}{\left(\omega^{n}\right)^{1 / n}\left(D_{\delta}^{n}\right)^{(n-1) / n}} \\
& \leq C^{\prime}\left(D_{\delta}^{n}\right)^{1 / n}+C^{\prime \prime} \frac{\left(\operatorname{Vol}(\alpha+\delta \omega)-D_{\delta}^{n}\right)^{1 / 2}}{\left(D_{\delta}^{n}\right)^{(n-1) / n}}
\end{aligned}
$$

However, since $\alpha \in \partial \mathcal{E}_{\mathrm{NS}}$, the class $\alpha$ cannot be big so

$$
\lim _{\delta \rightarrow 0} D_{\delta}^{n}=\operatorname{Vol}(\alpha)=0
$$

We can also take $D_{\delta}$ to approximate $\operatorname{Vol}(\alpha+\delta \omega)$ in such a way that $(\operatorname{Vol}(\alpha+\delta \omega)-$ $\left.D_{\delta}^{n}\right)^{1 / 2}$ tends to 0 much faster than $D_{\delta}^{n}$. Notice that $D_{\delta}^{n} \geq \delta^{n} \omega^{n}$, so in fact it is enough to take

$$
\operatorname{Vol}(\alpha+\delta \omega)-D_{\delta}^{n} \leq \delta^{2 n}
$$

This is the desired contradiction by (5.4).

As a corollary, we also get a solution of the "Hodge conjecture" for positive cones of $H^{n-1, n-1}(X)$, namely positive integral classes are generated by the corresponding cones of curves. This settles in the affirmative many of the conjectures made in [19]. 
Corollary 5.16. Let $X$ be a projective manifold. Then

(i) $\mathcal{N}_{\mathrm{NS}}=\overline{\mathrm{NE}(X)}$.

(ii) $\mathcal{M}_{\mathrm{NS}}=\overline{\operatorname{SME}(X)}=\overline{\mathrm{ME}(X)}$.

Proof. (i) is indeed (mostly) a standard result of algebraic geometry, a restatement of the fact that the cone of effective curves $\mathrm{NE}(X)$ is dual to the cone $\overline{\mathcal{K}_{\mathrm{NS}}}$ of nef divisors (see e.g. [24]) : clearly $\mathcal{N}_{\mathrm{NS}} \supset \overline{\mathrm{NE}(X)}=\mathcal{K}_{\mathrm{NS}}^{\vee}$, and the other direction $\mathcal{N}_{\mathrm{NS}} \subset \mathcal{K}_{\mathrm{NS}}^{\vee}$ is a consequence of $5.2(\mathrm{i})$.

(ii) It is obvious that $\operatorname{SME}(X) \subset \operatorname{ME}(X) \subset \mathcal{M}_{\mathrm{NS}} \subset\left(\mathcal{E}_{\mathrm{NS}}\right)^{\vee}$ (the latter inclusion follows from 5.2 (iii)). Now Theorem 5.14 implies $\left(\mathcal{E}_{\mathrm{NS}}\right)^{\vee}=\overline{\operatorname{SME}(X)}$, and (ii) follows.

Remark 5.17. If holomorphic Morse inequalities were known also in the Kähler case, we would infer by the same proof that " $\alpha$ not pseudo-effective" implies the existence of a blow-up $\mu: \widetilde{X} \rightarrow X$ and a Kähler metric $\widetilde{\omega}$ on $\widetilde{X}$ such that $\alpha$. $\mu_{\star}(\widetilde{\omega})^{n-1}<0$. In the special case when $\alpha=K_{X}$ is not pseudo-effective, we would expect the Kähler manifold $X$ to be covered by rational curves. The main trouble is that characteristic $p$ techniques are no longer available. On the other hand it is tempting to approach the question via techniques of symplectic geometry :

Question 5.18. Let $(M, \omega)$ be a compact real symplectic manifold. Fix an almost complex structure $J$ compatible with $\omega$, and for this structure, assume that $c_{1}(M) \cdot \omega^{n-1}>0$. Does it follow that $M$ is covered by rational $J$-pseudoholomorphic curves?

5.5. Applications and conjectures. The most important special case of Theorem 5.14 is

Theorem 5.19. If $X$ is a projective manifold and is not uniruled, then $K_{X}$ is pseudo-effective, i.e. $K_{X} \in \mathcal{E}_{\mathrm{NS}}$.

Proof. If $K_{X} \notin \mathcal{E}_{\mathrm{NS}}$, Prop. 5.2 shows that there is a moving curve $C_{t}$ such that $K_{X} \cdot C_{t}<0$. The standard "bend-and-break" lemma of Mori then implies that there is family $\Gamma_{t}$ of rational curves with $K_{X} \cdot \Gamma_{t}<0$, so $X$ is uniruled.

Of course, if the "abundance conjecture" is correct, the fact that $K_{X}$ is pseudoeffective would imply $\kappa(X) \geq 0$, and so every non uniruled variety shoud satisfy $\kappa(X) \geq 0$. This still seems beyond reach at the moment.

\section{Plurigenera and the Minimal Model Program}

In the case of algebraic surfaces, the Minimal Model Program (MMP) was already initiated by Italian geometers at the turn of the XXth century, and was finally completed by Zariski and Kodaira for all complex surfaces. The case of higher 
dimensions (starting with dimension 3) is a major endeavor of modern times, revitalized by Mori [41], Kawamata [29], [30], [31] and Shokurov [53], [54] among others (see also [33] for a good survey).

The basic question is to prove that every birational class of non uniruled algebraic varieties contains a "minimal" member $X$ exhibiting mild singularities ("terminal singularities"), where "minimal" is taken in the sense of avoiding unnecessary blow-ups; minimality actually means that $K_{X}$ is nef and not just pseudoeffective (pseudo-effectivity follows in general from Theorem 5.19). This requires performing certain birational transforms known as flips, and important questions are whether a) flips are indeed possible ("existence of flips"), b) the process terminates ("termination of flips"). Thanks to Kawamata [31] and Shokurov [53], [54], this has been proved in dimension 3 at the end of the 80's. Very recently, C. Hacon and J. McKernan [23] announced that flips exist in dimension $n$, if one assumes that a slightly stronger version of MMP (involving log pairs with real divisors) holds true in dimension $n-1$. As a consequence, the existence of flips obtained by Shokurov [55] in 2003 would be achieved in dimension 4 via a more systematic method. Strongly related to these issues are the following fundamental questions

(i) Finiteness of the canonical ring: is the canonical ring $R=\bigoplus H^{0}\left(X, m K_{X}\right)$ of a variety of general type always finitely generated?

If true, $\operatorname{Proj}(R)$ of this graded ring $R$ yields of course a "canonical model" in the birational class of $X$.

(ii) Boundedness of pluricanonical embeddings: is there a bound $r_{n}$ depending only on dimension $\operatorname{dim} X=n$, such that the pluricanonical map $\Phi_{m K_{X}}$ of a variety of general type yields a birational embedding in projective space for $m \geq r_{n}$ ?

(iii) Invariance of plurigenera: are plurigenera $p_{m}=h^{0}\left(X, m K_{X}\right)$ always invariant under deformation?

These questions involve taking "limits" of divisors as $m \rightarrow+\infty$, and therefore transcendental methods are a strong contender in the arena. Question (ii) was indeed solved in the affirmative by H. Tsuji [64], [63] under the assumption that the MMP program is solved, and in general by S. Takayama [61], and Ch. HaconJ. McKernan [22] by pursuing further Tsuji's ideas. Question (iii) was completely settled by Y.T. Siu ([58] in the case of varieties of general type, and [59] for arbitrary varieties). Quite recently, M. Păun gave a very elementary proof based merely on the Ohwawa-Takegoshi extension theorem, that we briefly sketch below. Y.T. Siu's work also gives strong support for the hope that (i) can be solved by a suitable combination of the $L^{2}$ existence theorems (Skoda's division theorem being one of the main ingredients). The following is a very slight extension of results by M. Păun [52] and B. Claudon [9], which are themselves based on the ideas of Y.T. Siu [59] and S. Takayama [62].

Theorem 6.1. Let $\pi: \mathcal{X} \rightarrow \Delta$ be a projective family over the unit disk, and let $\left(L_{j}, h_{j}\right)_{0 \leq j \leq m-1}$ be (singular) hermitian line bundles with semipositive curvature currents $i \Theta_{L_{j}, h_{j}} \geq 0$ on $\mathcal{X}$. Assume that 
(i) the restriction of $h_{j}$ to the central fiber $X_{0}$ is well defined (i.e. not identically $+\infty)$.

(ii) additionally the multiplier ideal sheaf $\mathcal{I}\left(h_{j \mid X_{0}}\right)$ is trivial for $1 \leq j \leq m-1$.

Then any section $\sigma$ of $\mathcal{O}\left(m K_{\mathcal{X}}+\sum L_{j}\right)_{\mid X_{0}} \otimes \mathcal{I}\left(h_{0 \mid X_{0}}\right)$ over the central fiber $X_{0}$ extends to $\mathcal{X}$.

We first state the technical version of the Ohsawa-Takegoshi $L^{2}$ extension theorem needed for the proof, which is a special case of Theorem 2.7 (see also Siu [59]).

Lemma 6.2. Let $\pi: \mathcal{X} \rightarrow \Delta$ be as before and let $(L, h)$ be a (singular) hermitian line bundle with semipositive curvature current $i \Theta_{L, h} \geq 0$ on $\mathcal{X}$. Let $\omega$ be a global Kähler metric on $\mathcal{X}$, and $d V_{\mathcal{X}}, d V_{X_{0}}$ the respective induced volume elements on $X_{0}$ and $\mathcal{X}$. Assume that $h_{X_{0}}$ is well defined. Then any holomorphic section $u$ of $\mathcal{O}\left(K_{\mathcal{X}}+L\right) \otimes \mathcal{I}\left(h_{\mid X_{0}}\right)$ extends into a section $\widetilde{u}$ over $\mathcal{X}$ satisfying an $L^{2}$ estimate

$$
\int_{\mathcal{X}}\|\widetilde{u}\|_{\omega \otimes h}^{2} d V_{\mathcal{X}} \leq C_{0} \int_{X_{0}}\|u\|_{\omega \otimes h}^{2} d V_{X_{0}}
$$

where $C_{0} \geq 0$ is some universal constant (independent of $\mathcal{X}, L, \ldots$ ).

Proof. We write $h_{j}=e^{-\varphi_{j}}$ in terms of local plurisubharmonic weights. Fix an auxiliary line bundle $A$ (which will later be taken to be sufficiently ample), and define inductively a sequence of line bundles $F_{p}$ by putting $F_{0}=A$ and

$$
F_{p}=F_{p-1}+K_{\mathcal{X}}+L_{r} \quad \text { if } p=m q+r, 0 \leq r \leq m-1 .
$$

By construction we have $F_{p+m}=F_{p}+m K_{\mathcal{X}}+\sum_{j} L_{j}$ and

$F_{0}=A, F_{1}=A+K_{\mathcal{X}}+L_{1}, \ldots, F_{p}=A+p K_{\mathcal{X}}+L_{1}+\ldots+L_{p}, 1 \leq p \leq m-1$.

The game is to construct inductively families of sections, say $\left(\widetilde{u}_{j}^{(p)}\right)_{j=1 \ldots N_{p}}$ of $F_{p}$ over $\mathcal{X}$ in such a way that

(a) for $p=0, \ldots, m-1, F_{p}$ is generated by its sections $\left(\widetilde{u}_{j}^{(p)}\right)_{j=1 \ldots N_{p}}$;

(b) we have the $m$-periodicity relations $N_{p+m}=N_{p}$ and $\widetilde{u}_{j}^{(p)}$ is an extension of $u_{j}^{(p)}:=\sigma^{q} u_{j}^{(r)}$ over $\mathcal{X}$ for $p=m q+r$, where $u_{j}^{(r)}:=\widetilde{u}_{j \mid X_{0}}^{(r)}, 0 \leq r \leq m-1$.

Property (a) can certainly be achieved by taking $A$ ample enough so that $F_{0}, \ldots$, $F_{m-1}$ are generated by their sections, and by choosing the $\widetilde{u}_{j}^{(p)}$ appropriately for $p=0, \ldots, m-1$. Now, by induction, we equip $F_{p-1}$ with the tautological metric $|\xi|^{2} / \sum\left|\widetilde{u}_{j}^{(p-1)}(x)\right|^{2}$, and $F_{p}-K_{\mathcal{X}}=F_{p-1}+L_{r}$ with that metric multiplied by $h_{r}=e^{-\varphi_{r}}$; it is clear that these metrics have semipositive curvature currents (the metric on $F_{p}$ itself if obtained by using a smooth Kähler metric $\omega$ on $\mathcal{X}$ ). In this setting, we apply the Ohsawa-Takegoshi theorem to the line bundle $F_{p-1}+L_{r}$ to 
extend $u_{j}^{(p)}$ into a section $\widetilde{u}_{j}^{(p)}$ over $\mathcal{X}$. By construction the pointwise norm of that section in $F_{p \mid X_{0}}$ in a local trivialization of the bundles involved is the ratio

$$
\frac{\left|u_{j}^{(p)}\right|^{2}}{\sum_{\ell}\left|u_{\ell}^{(p-1)}\right|^{2}} e^{-\varphi_{r}}
$$

up to some fixed smooth positive factor depending only on the metric induced by $\omega$ on $K_{\mathcal{X}}$. However, by the induction relations, we have

$$
\frac{\sum_{j}\left|u_{j}^{(p)}\right|^{2}}{\sum_{\ell}\left|u_{\ell}^{(p-1)}\right|^{2}} e^{-\varphi_{r}}= \begin{cases}\frac{\sum_{j}\left|u_{j}^{(r)}\right|^{2}}{\sum_{\ell}\left|u_{\ell}^{(r-1)}\right|^{2}} e^{-\varphi_{r}} & \text { for } p=m q+r, 0<r \leq m-1, \\ \frac{\sum_{j}\left|u_{j}^{(0)}\right|^{2}}{\sum_{\ell}\left|u_{\ell}^{(m-1)}\right|^{2}}|\sigma|^{2} e^{-\varphi_{0}} & \text { for } p \equiv 0 \bmod m .\end{cases}
$$

Since the sections $\left(u_{j}^{(r)}\right)$ generate their line bundle, the ratios involved are positive functions without zeroes and poles, hence smooth and bounded [possibly after shrinking the base disc $\Delta$, as is permitted]. On the other hand, assumption (ii) and the fact that $\sigma$ has coefficients in the multiplier ideal sheaf $\mathcal{I}\left(h_{0 \mid X_{0}}\right)$ tell us that $e^{-\varphi_{r}}, 1 \leq r<m$ and $|\sigma|^{2} e^{-\varphi_{0}}$ are locally integrable on $X_{0}$. It follows that there is a constant $C_{1} \geq 0$ such that

$$
\int_{X_{0}} \frac{\sum_{j}\left|u_{j}^{(p)}\right|^{2}}{\sum_{\ell}\left|u_{\ell}^{(p-1)}\right|^{2}} e^{-\varphi_{r}} d V_{\omega} \leq C_{1}
$$

for all $p \geq 1$ [of course, the integral certainly involves finitely many trivializations of the bundles involved, whereas the integrand expression is just local in each chart]. Inductively, the $L^{2}$ extension theorem produces sections $\widetilde{u}_{j}^{(p)}$ of $F_{p}$ over $\mathcal{X}$ such that

$$
\int_{\mathcal{X}} \frac{\sum_{j}\left|\widetilde{u}_{j}^{(p)}\right|^{2}}{\sum_{\ell}\left|\widetilde{u}_{\ell}^{(p-1)}\right|^{2}} e^{-\varphi_{r}} d V_{\omega} \leq C_{2}=C_{0} C_{1} .
$$

The next idea is to extract the limits of $p$-th roots of these sections to get a singular hermitian metric on $m K_{\mathcal{X}}+\sum L_{j}$. As the functions $e^{-\varphi_{r}}$ are locally bounded below $\left(\varphi_{r}\right.$ being psh), the Hölder inequality implies that

$$
\int_{\mathcal{X}}\left(\sum_{j}\left|\widetilde{u}_{j}^{(p)}\right|^{2}\right)^{1 / p} d V_{\omega} \leq C_{3}
$$

Jensen's inequality together with well known facts of potential theory now show that some subsequence of the sequence of plurisubharmonic functions $\frac{1}{q} \log \sum_{j}\left|\widetilde{u}_{j}^{(m q)}\right|^{2}$ [which should be thought of as weights on the $\mathbb{Q}$-line bundles $\left.\frac{1}{q}\left(A+q\left(m K_{\mathcal{X}}+\sum L_{j}\right)\right)\right]$ converges almost everywhere to the weight $\psi$ of a singular hermitian metric $H$ with semi-positive curvature on $m K_{\mathcal{X}}+\sum L_{j}$, in the form of an upper regularized limit

$$
\psi(z)=\limsup _{\zeta \rightarrow z} \lim _{\nu \rightarrow+\infty} \frac{1}{q_{\nu}} \log \sum_{j}\left|\widetilde{u}_{j}^{\left(m q_{\nu}\right)}(\zeta)\right|^{2} .
$$


On $X_{0}$ we have

$$
\lim _{q \rightarrow+\infty} \frac{1}{q} \log \sum_{j}\left|u_{j}^{(m q)}\right|^{2}=\lim _{q \rightarrow+\infty} \frac{1}{q} \log \left(|\sigma|^{2 q} \sum_{j}\left|u_{j}^{(0)}\right|^{2}\right)=\log |\sigma|^{2},
$$

hence $\psi(z) \geq \log |\sigma|^{2}$ and $\|\sigma\|_{H} \leq 1$. We equip the bundle

$$
G=(m-1) K_{\mathcal{X}}+\sum L_{j}
$$

with the metric $\gamma=H^{1-1 / m} \prod h_{j}^{1 / m}$, and $m K_{\mathcal{X}}+\sum L_{j}=K_{\mathcal{X}}+G$ with the metric $\omega \otimes \gamma$. Clearly $\gamma$ has a semipositive curvature current on $\mathcal{X}$ and in a local trivialization we have

$$
\|\sigma\|_{\omega \otimes \gamma}^{2} \leq C|\sigma|^{2} \exp \left(-\left(1-\frac{1}{m}\right) \psi+\frac{1}{m} \sum \varphi_{j}\right) \leq C\left(|\sigma|^{2} \prod e^{-\varphi_{j}}\right)^{1 / m}
$$

on $X_{0}$. Since $|\sigma|^{2} e^{-\varphi_{0}}$ and $e^{-\varphi_{r}}, r>0$ are all locally integrable, we see that $\|\sigma\|_{\omega \otimes \gamma}^{2}$ is also locally integrable on $X_{0}$ by the Hölder inequality. A new (and final) application of the $L^{2}$ extension theorem to the hermitian line bundle $(G, \gamma)$ implies that $\sigma$ can be extended to $\mathcal{X}$. The theorem is proved.

The special case of the theorem obtained by taking all bundles $L_{j}$ trivial tells us in particular that any pluricanonical section $\sigma$ of $m K_{\mathcal{X}}$ over $X_{0}$ extends to $\mathcal{X}$. By the upper semi-continuity of $t \mapsto h^{0}\left(X_{t}, m K_{X_{t}}\right)$, this implies

Corollary 6.3. (Siu [59]) For any projective family $t \mapsto X_{t}$ of algebraic varieties, the plurigenera $p_{m}\left(X_{t}\right)=h^{0}\left(X_{t}, m K_{X_{t}}\right)$ do not depend on $t$.

At the moment, it should be observed that there are no purely algebraic proofs of the invariance of plurigenera, though Y. Kawamata [32] has given an algebraic proof in the case of varieties of general type.

\section{References}

[1] Andreotti, A., Vesentini, E., Carleman estimates for the Laplace-Beltrami equation in complex manifolds, Publ. Math. I.H.E.S. 25 (1965) 81-130.

[2] Biswas, I., Narasimhan, M.S., Hodge classes of moduli spaces of parabolic bundles over the general curve, J. Algebraic Geom. 6 (1997), 697-715.

[3] Bochner, S., Curvature and Betti numbers (I) and (II), Ann. of Math. 49 (1948) 379-390; 50 (1949) 77-93.

[4] Boucksom, S., Cônes positifs des variétés complexes compactes. Thesis, Grenoble 2002.

[5] Boucksom, S., Demailly, J.P., Păun, M., Peternell, Th., The pseudo-effective cone of a compact Kähler manifold and varieties of negative Kodaira dimension, arXiv: math.AG/0405285. 
[6] Buchdahl, N., On compact Kähler surfaces, Ann. Inst. Fourier 49 (1999) 287-302.

[7] Buchdahl, N., A Nakai-Moishezon criterion for non-Kähler surfaces, Ann. Inst. Fourier 50 (2000) 1533-1538.

[8] Campana, F., Peternell, Th., Geometric stability of the cotangent bundle and the universal cover of a projective manifold, arXiv: math.AG/0405093.

[9] Claudon, B., Invariance for multiples of the twisted canonical bundle, Ann. Inst. Fourier 57 (2007), 289-300.

[10] Demailly, J.-P., Estimations $L^{2}$ pour l'opérateur $\bar{\partial}$ d'un fibré vectoriel holomorphe semi-positif au-dessus d'une variété kählérienne complète, Ann. Sci. École Norm. Sup. 4e Sér. 15 (1982) 457-511.

[11] Demailly, J.-P., Champs magnétiques et inégalités de Morse pour la $d^{\prime \prime}$-cohomologie, Ann. Inst. Fourier 35 (1985), 189-229.

[12] Demailly, J.-P., Transcendental proof of a generalized Kawamata-Viehweg vanishing theorem, C. R. Acad. Sci. Paris Sér. I Math. 309 (1989) 123-126 and: Proceedings of the Conference "Geometrical and algebraical aspects in several complex variables" held at Cetraro, Univ. della Calabria, June 1989, 81-94.

[13] Demailly, J.-P., Singular hermitian metrics on positive line bundles, Proceedings of the Bayreuth conference "Complex algebraic varieties", April 2-6, 1990, edited by K. Hulek, T. Peternell, M. Schneider, F. Schreyer, Lecture Notes in Math. n ${ }^{\circ} 1507$, Springer-Verlag, 1992, 87-104.

[14] Demailly, J.-P., Regularization of closed positive currents and intersection theory, $J$. Algebraic Geometry 1 (1992), 361-409.

[15] Demailly, J.-P., A numerical criterion for very ample line bundles, J. Differential Geom. 37 (1993) 323-374.

[16] Demailly, J.-P., Multiplier ideal sheaves and analytic methods in algebraic geometry, Lecture Notes, School on "Vanishing theorems and effective results in Algebraic Geometry, ICTP Trieste, April 2000, Publications of ICTP, 2001.

[17] Demailly, J.-P., Ein, L., Lazarsfeld, R., A subadditivity property of multiplier ideals, arXiv: math.AG/0002035, Michigan Math. J., special volume in honor of William Fulton, 48 (2000), 137-156.

[18] Demailly, J.-P., Păun, M., Numerical characterization of the Kähler cone of a compact Kähler manifold, arXiv: math.AG/0105176, Annals of Math. 159 (2004) 12471274 .

[19] Demailly, J.-P.; Peternell, Th.; Schneider, M., Holomorphic line bundles with partially vanishing cohomology, Israel Math. Conf. Proc. vol. 9 (1996) 165-198.

[20] Demailly, J.-P., Peternell, Th.; Schneider, M., Pseudo-effective line bundles on compact Kähler manifolds, Intern. J. Math. 6 (2001) 689-741.

[21] Fujita, T., Approximating Zariski decomposition of big line bundles, Kodai Math. J. 17 (1994), 1-3.

[22] Hacon, Ch., McKernan, J., Boundedness of pluricanonical maps of varieties of general type, arXiv: math.AG/0504327, Invent. Math. 166 (2006), 1-25

[23] Hacon, Ch., McKernan, J., On the existence of flips, arXiv: math.AG/0507597.

[24] Hartshorne, R., Ample subvarieties of algebraic varieties, Lecture Notes in Math. no 156, Springer-Verlag, Berlin (1970). 
[25] Hörmander, L., $L^{2}$ estimates and existence theorems for the $\bar{\partial}$ operator, Acta Math. 113 (1965) 89-152.

[26] Huybrechts, D., Compact Hyperkähler Manifolds: Basic Results, Invent. Math. 135 (1999), 63-113.

[27] Huybrechts, D., Compact Hyperkähler Manifolds: Basic Results, II (The Projectivity Criterion for Hyperkähler manifolds as a Consequence of the Demailly-Păun Theorem), Invent. Math. 152 (2003), 209-212.

[28] Kawamata, Y., A generalization of Kodaira-Ramanujam's vanishing theorem, Math. Ann. 261 (1982) 43-46.

[29] Kawamata, Y., The cone of curves of algebraic varieties, Ann. of Math. 119 (1984), 603-633.

[30] Kawamata, Y., Minimal models and the kodaira dimension of algebraic fiber spaces, J. reine Angew. Math. 363 (1985), 1-46.

[31] Kawamata, Y., Termination of log flips for algebraic 3-folds, Internat. J. Math. 3 (1992), 653-659.

[32] Kawamata, Y., Deformation of canonical singularities, J. Amer. Math. Soc., 12 (1999), 85-92.

[33] Kawamata, Y., Matsuda, K., Matsuki, K., Introduction to the minimal model program, Adv. Stud. Pure Math. 10 (1987) 283-360.

[34] Kleiman, S., Toward a numerical theory of ampleness, Ann. Math. 84 (1966), 293344.

[35] Kodaira, K., On Kähler varieties of restricted type, Ann. of Math. 60 (1954) 28-48.

[36] Kohn, J.J., Harmonic integrals on strongly pseudo-convex manifolds I, Ann. Math. (2), 78 (1963) 206-213.

[37] Kodaira, K., Spencer, D.C., On deformations of complex analytic structures. III. Stability theorems for complex structures, Ann. of Math. 71 (1960) 43-76.

[38] Lamari, A., Courants kählériens et surfaces compactes, Ann. Inst. Fourier 49 (1999) 263-285.

[39] Lamari, A., Le cone Kählérien d'une surface, J. Math. Pures Appl. 78 (1999) 249263.

[40] Manivel, L., Un théorème de prolongement $L^{2}$ de sections holomorphes d'un fibré vectoriel, Math. Zeitschrift 212 (1993) 107-122.

[41] Mori, S., Threefolds whose canonical bundles are not numerically effective, Ann. of Math. 116 (1982), 133-176.

[42] Nadel, A.M., Multiplier ideal sheaves and Kähler-Einstein metrics of positive scalar curvature, Proc. Nat. Acad. Sci. U.S.A. 86 (1989) -7299-7300 and Annals of Math. 132 (1990), 549-596.

[43] Ohsawa, T., On the extension of $L^{2}$ holomorphic functions, II, Publ. RIMS, Kyoto Univ. 24 (1988) 265-275.

[44] Ohsawa, T., On the extension of $L^{2}$ holomorphic functions, III : negligible weights, Math. Zeitschrift 219 (1995) 215-225. 
[45] Ohsawa, T., On the extension of $L^{2}$ holomorphic functions, IV: A new density concept, Mabuchi, T. (ed.) et al., Geometry and analysis on complex manifolds, Festschrift for Professor S. Kobayashi's 60th birthday. Singapore: World Scientific, (1994) 157-170.

[46] Ohsawa, T., On the extension of L2 holomorphic functions, V, Effects of generalization, Nagoya Math. J. 161 (2001), 1-21.

[47] Ohsawa, T., On the extension of $L^{2}$ holomorphic functions, VI, A limiting case, Explorations in complex and Riemannian geometry, Contemp. Math., 332, Amer. Math. Soc., Providence, RI (2003) 235-239.

[48] Ohsawa, T., Takegoshi, K., On the extension of $L^{2}$ holomorphic functions, Math. Zeitschrift, 195 (1987) 197-204.

[49] Păun, M., Sur l'effectivité numérique des images inverses de fibrés en droites, Math. Ann. 310 (1998), 411-421.

[50] Păun, M., Fibré en droites numériquement effectifs et variétés Kählériennes compactes à courbure de Ricci nef, Thèse, Université de Grenoble 1 (1998), 80 p.

[51] Păun, M., Semipositive (1,1)-cohomology classes on projective manifolds, Preprint de l'I.R.M.A., Strasbourg.

[52] Păun, M., Siu's invariance of plurigenera : a one-tower proof, preprint November 2005.

[53] Shokurov, V. V., Extremal contractions of three-dimensional algebraic varieties, (Russian), Yaroslavl Gos. Ped. Inst., 102 (1983), 74-90.

[54] Shokurov, V. V., 3-fold log models, Algebraic Geometry, 4, J. Math. Sci. 81 (1996), 2667-2699.

[55] Shokurov, V. V., Prelimiting flips, Proc. Steklov Inst. of Math. 240 (2003), 82-219.

[56] Siu, Y.-T., Analyticity of sets associated to Lelong numbers and the extension of closed positive currents, Invent. Math. 27 (1974), 53-156.

[57] Siu, Y.-T., Some recent results in complex manifold theory for the semi-positive case, survey article in the Proceedings of the Mathematisches Arbeitstagung, Bonn 1984.

[58] Siu, Y.-T., Invariance of plurigenera, Invent. Math. 134 (1994) 631-639.

[59] Siu, Y.-T., Extension of twisted pluricanonical sections with plurisubharmonic weight and invariance of semipositively twisted plurigenera for manifolds not necessarily of general type, Complex Geometry (Göttingen, 2000), Springer, Berlin, 2002, $223-277$.

[60] Skoda, H., Morphismes surjectifs de fibrés vectoriels semi-positifs, Ann. Sci. Ecole Norm. Sup. 11 (1978) 577-611.

[61] Takayama, S., Pluricanonical systems on algebraic varieties of general type, Invent. Math. 165 (2006) 551-587.

[62] Takayama, S., On the invariance and lower semi-continuity of plurigenera of algebraic varieties, J. Algebraic Geom. 16 (2007) 1-18.

[63] Tsuji, H., Subadjunction theorem, arXiv: math.AG/0111311, Complex analysis in several variables - Memorial Conference of Kiyoshi Oka's Centennial Birthday, 313318, Adv. Stud. Pure Math. 42, Math. Soc. Japan, Tokyo, 2004.

[64] Tsuji, H., Pluricanonical systems of projective varieties of general type, arXiv: math.AG/9909021, Osaka J. of Mathematics 43 (2006) 967-995. 
[65] Viehweg, E., Vanishing theorems, J. Reine Angew. Math. 335 (1982) 1-8.

[66] Voisin, C., A counterexample to the Hodge conjecture extended to Kähler varieties, Int. Math. Res. Not. 20 (2002) 1057-1075.

[67] Voisin, C., On the homotopy types of compact Kähler and complex projective manifolds Invent. Math. 157 (2004), 329-343.

[68] Voisin, C., On the homotopy types of Kähler manifolds and the birational Kodaira problem, J. Differential Geom. 72 (2006), 43-71.

[69] Yau, S.-T., On the Ricci curvature of a complex Kähler manifold and the complex Monge-Ampère equation, Comm. Pure Appl. Math. 31 (1978), 339-411.

[70] Zucker, S., The Hodge conjecture for cubic fourfolds, Compositio Math. 34 (1977) 199-209.

Université de Grenoble I, Institut Fourier, 100 rue des Maths, BP 74, 38402 SaintMartin d'Hères

E-mail: demailly@fourier.ujf-grenoble.fr 